\title{
Cell Interplay in Osteoarthritis
}

\author{
Zihao Li', Ziyu Huang ${ }^{2}$ and Lunhao Bai ${ }^{1 *}$ \\ ${ }^{1}$ Department of Orthopedics, Shengjing Hospital of China Medical University, Shenyang, China, ${ }^{2}$ Foreign Languages \\ College, Shanghai Normal University, Shanghai, China
}

Osteoarthritis $(\mathrm{OA})$ is a common chronic disease and a significant health concern that needs to be urgently solved. OA affects the cartilage and entire joint tissues, including the subchondral bone, synovium, and infrapatellar fat pads. The physiological and pathological changes in these tissues affect the occurrence and development of OA. Understanding complex crosstalk among different joint tissues and their roles in OA initiation and progression is critical in elucidating the pathogenic mechanism of OA. In this review, we begin with an overview of the role of chondrocytes, synovial cells (synovial fibroblasts and macrophages), mast cells, osteoblasts, osteoclasts, various stem cells, and engineered cells (induced pluripotent stem cells) in OA pathogenesis. Then, we discuss the various mechanisms by which these cells communicate, including paracrine signaling, local microenvironment, co-culture, extracellular vesicles (exosomes), and

OPEN ACCESS

Edited by:

Kazunori Shimomura

Osaka University, Japan

Reviewed by:

Csaba Matta,

University of Debrecen, Hungary Janos Kanczler,

University of Southampton,

United Kingdom

*Correspondence:

Lunhao Bai

lunhaobai_ace@163.com

Specialty section:

This article was submitted to

Stem Cell Research,

a section of the journal

Frontiers in Cell and Developmental

Biology

Received: 04 June 2021

Accepted: 14 July 2021

Published: 03 August 2021

Citation:

Li Z, Huang Z and Bai L (2021)

Cell Interplay in Osteoarthritis.

Front. Cell Dev. Biol. 9:720477.

doi: 10.3389/fcell.2021.720477 cell tissue engineering. We particularly focus on the therapeutic potential and clinical applications of stem cell-derived extracellular vesicles, which serve as modulators of cell-to-cell communication, in the field of regenerative medicine, such as cartilage repair. Finally, the challenges and limitations related to exosome-based treatment for OA are discussed. This article provides a comprehensive summary of key cells that might be targets of future therapies for OA.

Keywords: cartilage, subchondral bone, synovium, infrapatellar fat pad, stem cell, exosome, osteoarthritis

\section{INTRODUCTION}

Osteoarthritis (OA) is a common degenerative disease of the joints that causes chronic pain and motor dysfunction and affects the quality of life of more than 300 million people worldwide (GBD 2017 Risk Factor Collaborators, 2018). OA also poses a considerable economic burden on patients and is a major public health problem. OA's current treatment strategies include non-drug treatment (e.g., exercise, weight reduction, and physiotherapy), drug treatment, and surgery (Bannuru et al., 2019; Kloppenburg and Berenbaum, 2020). Non-pharmacological therapies are used for patients in the early stages of OA to delay its development. However, the effects of these approaches on early symptoms are limited, particularly on structural diseases (Cutolo et al., 2015). On the other hand, medications, including pain relievers and non-steroidal anti-inflammatory drugs, are prescribed to control the pain, preserve functional capacity, and improve the quality of daily life. However, because patients with $\mathrm{OA}$ are prone to complications, inappropriate drug treatment and multidrug therapy can increase the risk of side effects (Gore et al., 2011). Meanwhile, surgical treatment is considered for patients with advanced OA; however, this modality is associated with high failure rates and complications, and additional costs. Because the molecular mechanisms underlying OA initiation and progression remain poorly understood, there are no current interventions with 
satisfactory curative effects that can delay disease progression (Dieppe et al., 2011; Bannuru et al., 2019). Therefore, new insights into the mechanism of OA pathogenesis are required to promote the development of new therapies that meet future clinical needs.

Osteoarthritis was previously considered to be caused by mechanical damage or the habitual overuse of a joint that is an inevitable part of aging. However, it has become increasingly clear that $\mathrm{OA}$ is much more complex than a wear-and-tear disease, and various factors such as inflammation, metabolism, and biochemical machinery play an important role in its pathogenesis (Brandt et al., 2006; Martel-Pelletier et al., 2016). Furthermore, aging, obesity, joint damage, and high-intensity activities have been identified as risk factors leading to OA development (Zhang and Jordan, 2008; Silverwood et al., 2015; Martel-Pelletier et al., 2016). Hence, OA is now viewed as a multifactorial disease that involves local and systemic factors and has multiple pathogenetic mechanisms. Therefore, these factors must be considered when exploring new treatment methods for OA.

In addition, rather than merely involving the destruction of articular cartilage, OA is now more accurately thought of as a disease of the whole joint and is characterized by the partial loss of cartilage, thickening of the synovial sac, subchondral bone sclerosis and osteophyte formation, and changes in the structure of joints, ligaments, and surrounding muscles (Pereira et al., 2011; Hunter and Bierma-Zeinstra, 2019). During its development, different tissues within the joint and their interactions contribute to the pathology and clinical symptoms of OA (Burr, 1998; de Lange-Brokaar et al., 2012). Recently, the role of the subchondral bone, which refers to the cortical bone layer under the articular cartilage and the trabecular bone in the lower part of the joint, in OA pathogenesis has attracted increasing attention. Studies have shown that the subchondral bone may affect cartilage degeneration through changes in mechanical stress or paracrine-mediated interaction between the bone and cartilage (Sharma et al., 2013; Zhen et al., 2013; Lin et al., 2019). In an inflammatory environment, synovial fibroblasts (SFB) may affect the formation of osteophytes and the degradation of the cartilage matrix by releasing bone regulatory factors (including BMP-2) and pro-inflammatory factors (such as IL1ß) (Mathiessen and Conaghan, 2017). Infrapatellar fat pad (IPFP) and synovium can also release various pro-inflammatory mediators during inflammation. These mediators not only result in the abnormal structure and function of synovial tissue but also aggravate cartilage damage and the development of OA (Clockaerts et al., 2010; Mathiessen and Conaghan, 2017; Kuang et al., 2020). Therefore, new insights into the interaction and communication among the different cells in the joint may lead to a greater understanding of the disease mechanism of $\mathrm{OA}$ and provide new perspectives for the development of OA treatment strategies.

In this review, we first introduce the cells found in joint tissues and their role in OA pathogenesis. Then, we discuss the various ways by which these cells communicate, including paracrine signaling, local microenvironment, co-culture, extracellular vesicles (EVs), and exosomes. Finally, we summarize the recent studies on the therapeutic potential and clinical applications of stem cell-derived EVs for OA treatment. This article provides a comprehensive summary of key cells that might be targets of future therapies for OA.

\section{JOINT CELLS AND TISSUES}

\section{Cartilage and Chondrocytes}

Articular cartilage is the connective tissue located on the surface of the synovial joint and plays a role in lubrication and weight-bearing during joint activities. Due to the lack of a vascular system and limited oxygen and nutrient supply, articular cartilage has low regenerative potential (Sophia Fox et al., 2009; Chen et al., 2017; Singh et al., 2019). In addition to the chondrocytes, which were long considered to be the only cell type within articular cartilage, cartilage stem/progenitor cells (CSPC) have been recently identified in OA cartilage (Williams et al., 2010), representing approximately $10 \%$ of the total cells (Alsalameh et al., 2004; Bosserhoff et al., 2014; Riegger et al., 2018). Moreover, single-cell RNAseq analysis revealed various chondrocyte populations in advanced $\mathrm{OA}$, including homeostatic chondrocytes, proliferative chondrocytes, effector chondrocytes, regulatory chondrocytes, pre-hypertrophic chondrocytes, hypertrophic chondrocytes, and fibrocartilage chondrocytes (Ji et al., 2019).

As the resident cell type in tissues, chondrocytes can form an extracellular matrix mainly composed of aggrecan and type II collagen. However, chondrocytes only account for $1-5 \%$ of the total volume of cartilage tissue (Bhosale and Richardson, 2008). Owing to the limited number of cells and their pyknotic nature, damage caused by various risk factors, such as abnormal mechanical load, trauma, and inflammation, can lead to changes in the structure and function of cartilage. Joint degenerative diseases are prone to occur without timely and adequate treatment (Palazzo et al., 2016; Vina and Kwoh, 2018; Li et al., 2019; Singh et al., 2019). Although the composition of articular cartilage is simple, its horizontal-layered structure containing chondrocytes with various morphologies and different distribution and secretion characteristics remains well-organized.

Changes in the metabolic state of chondrocytes lead to the imbalance of collagen synthesis and degradation, cartilage degeneration, chondrosenescence, and an intra-articular inflammatory environment, ultimately leading to OA (Martin and Buckwalter, 2002). The metabolic changes in chondrocytes may exhibit distinct characteristics and have been described in four clinical OA phenotypes. First, the inflammationassociated OA phenotype is characterized by a low degree of inflammation (Scanzello, 2017). The continuous accumulation of pro-inflammatory mediators leads to the excessive production of reactive oxygen species and mtDNA damage and drives the catabolic reaction in the chondrocytes, subsequently disrupting the balance between cartilage repair and damage. The second phenotype is the mechanical overload-associated OA phenotype. Normal physiological load is important to maintain chondrocyte function and ECM metabolic balance. Mechanical overloading is thus harmful to chondrocytes and results in a weakened anabolic response, decreased extracellular matrix (ECM) synthesis, and 
enhanced catabolic response, thereby stimulating the synthesis of matrix metalloproteinases (MMPs) (Loeser et al., 2012). Metabolic syndrome-associated OA phenotype is characterized by increased fasting plasma glucose concentration, increased triglyceride level, decreased high-density lipoprotein level, and/or hypertension (Dickson et al., 2019). The occurrence and development of OA can be positively affected by increasing the production of fat-derived pro-inflammatory mediators, such as advanced glycation end-products (Oren et al., 2011). The last is the aging-associated OA phenotype. The catabolism of aging chondrocytes is active, and disruption of the interplay between autophagy and the inflammasome is observed in an inflammatory environment (Salminen et al., 2012). In addition, the decline in chondrocyte mitochondrial function is accompanied by decreased chondrocyte autophagy and increased apoptosis (Blanco et al., 2018). The proliferation and synthesis ability of chondrocytes in aging articular cartilage also decreases; however, their ability to produce pro-inflammatory mediators and MMPs remains unchanged (Loeser, 2009). Overall, the initiation and progression of $\mathrm{OA}$ are closely related to the phenotypic changes in chondrocytes.

\section{Synovium and Synovial Cells}

Increasing evidence has shown that the mutual communication between different tissues in the joint is essential for maintaining joint homeostasis. The communication between synovium and cartilage not only contributes to OA symptoms but is also a key factor in disease pathogenesis. The morphology and cell composition of the synovium are often used as biomarkers for the development of OA. Although the synovial tissue may not be affected in the early-stage of OA, many patients with advanced OA suffer from severe synovitis (Wenham and Conaghan, 2010).

In healthy joints, the synovium is mainly composed of two types of synovial cells. Type A macrophage-like synovial cells, which are relatively small, mainly have a phagocytic function and produce pro-inflammatory cytokines. Meanwhile, type B fibroblast-like synovial cells provide structure, nutrition, and lubrication and represent approximately $75 \%$ of the cells in the synovium (Firestein and McInnes, 2017). In addition, fibroblastslike synovial cells can migrate to the site of tissue remodeling and interact with ECM molecules via specific surface receptors (Pap and Bertrand, 2012). They can perceive and respond to the changes in the composition and structure of the surrounding synovial tissue by adjusting their interaction with and the production of ECM components. In the initiation of OA, the intimal lining layer becomes hypertrophic and is infiltrated by macrophages, fibroblasts, mast cells, $\mathrm{T}$ cells, $\mathrm{B}$ cells, dendritic cells, and neutrophils, leading to a 5- to 10-fold increase in cell density (Culemann et al., 2019). These infiltrating cells promote the production of pro-inflammatory cytokines and catabolites, thereby changing the composition of synovial fluid, an important source of these pro-inflammatory mediators in OA (Falconer et al., 2018). In the synovial fluid of OA patients, the proportion of macrophages is relatively low, whereas that of mast cells is relatively high (de Lange-Brokaar et al., 2012; Robinson et al., 2016; Xie et al., 2019).

\section{Macrophages}

Synovitis can occur at both the early and late stages of OA (Sellam and Berenbaum, 2010) and is characterized by the accumulation of macrophages in the intimal lining layer (Sun A. R. et al., 2016). Macrophages are heterogeneous and plastic immune cells that produce chemokines and cytokines in inflamed joints. Macrophages can be activated by various stimuli, including proinflammatory (IL-1 $\beta$, TNF- $\alpha$, and IL-6) and immunomodulatory cytokines (IL-4 and IL-10) (Wang et al., 2014; Dutta et al., 2020) and abnormal mechanical forces, which are mostly produced during stress or cell damage (Liu et al., 2018). Activated macrophages are generally classified into two distinct phenotypes, namely, classically activated/inflammatory (M1) type and alternatively activated/immunomodulatory (M2) type.

Polarized M1 macrophages can generate a large amount of pro-inflammatory cytokines, nitric oxide (NO), and reactive oxygen species, thereby enhancing host defense response (Mosser, 2003; Genin et al., 2015). However, excessive activation of M1 macrophages can lead to autoimmune diseases and tissue damage (Mosser and Edwards, 2008). M2 macrophages are mainly present in the subsiding phase of inflammation and are responsible for producing anti-inflammatory cytokines and eliminating apoptotic cells. In addition, M2 macrophages can produce osteogenic growth factors, such as bone morphogenetic protein 2 (BMP-2; an effective promoter of osteogenic differentiation of MSCs belonging to a subclass of the TGF- $\beta$ family), (Champagne et al., 2002; Li C. J. et al., 2018) TGF- $\beta$, (Assoian et al., 1987) 1,25-dihydroxy vitamin D3, (Kreutz et al., 1993) and osteopontin (Takahashi et al., 2004).

Therefore, the imbalance in the ratio between these two kinds of cells in OA may be related to the initiation and progression of OA (Xue et al., 2019). The significantly increased number of M1 macrophages in synovial tissues (Sun et al., 2017) and the high proportion of M2 macrophages have clinical diagnostic significance for OA (Chen et al., 2020). However, it is difficult to assess the polarization state of synovial macrophages before OA occurs or even in its early stages. In late-stage OA, M1and M2-like macrophages can coexist in the joint synovium and adjacent adipose tissues. However, the role of macrophages in OA initiation and progression is unquestionable. Macrophages play an important role in the occurrence of OA through inflammatory factors, cytokines, and proteins, whether it is inflammatory or mechanical injury (Bondeson et al., 2010).

\section{Mast Cells}

Mast cells (MCs), a type of immune cells that reside in tissues, play a pivotal role in allergic reactions (Galli and Tsai, 2012). The synovial fluid of patients with OA showed an increased number of MCs and increased concentration of certain MC mediators, such as histamine and tryptase (Bridges et al., 1991) (Buckley et al., 1997). The role of MCs in bone metabolism remains controversial (Urist and McLean, 1957). In MC-deficient mouse models, MCs were involved in the occurrence and development of OA (Schubert et al., 2015; Kroner et al., 2017) and fracture healing and may also be involved in regulating the production of osteoclasts (Behrends et al., 2014; Kroner et al., 2017). Many MC mediators can regulate or 
induce bone metabolism by inhibiting osteoblast activity (such as IL-1, TNF) and/or promoting osteoclastogenesis (such as histamine, TNF, IL-6) (Biosse-Duplan et al., 2009; Pietschmann et al., 2016). MCs can also play a role in maintaining bone homeostasis. For instance, the transforming growth factor- $\beta$ (TGF- $\beta$ ) can stimulate the production of osteoblasts, while IL-12 and interferon- $\gamma($ IFN- $\gamma)$ can inhibit the formation of osteoclasts (Pietschmann et al., 2016).

Several clinical studies have reported the increased expression of genes involved in MC differentiation and activity in the synovial tissues of OA patients (Wang et al., 2019). MCdeficient mice were protected from inflammation and cartilage destruction of OA; however, MC implantation in engraftment reversed this protection (Wang et al., 2019). In addition, the inhibition of tryptase activity in wild-type mice reduced the concentration of pro-inflammatory mediators, such as IL-6, IL$1 \beta$, IL- 8 , and MMP-3. Furthermore, the synovial MCs of patients with OA can secrete TNF- $\alpha$ following stimulation with the highaffinity receptor of IgG (Lee et al., 2013). In a cross-sectional cohort study, $\mathrm{H} 1$ antihistamine treatment was associated with decreased prevalence of OA (Shirinsky and Shirinsky, 2018). These results confirm the critical role of MC in the occurrence and development of $\mathrm{OA}$ and indicate that MCs may be a potential therapeutic target for OA.

\section{Subchondral Bone and Osteoblasts and Osteoclasts}

The degeneration and degradation of articular cartilage had long been considered the leading cause of $\mathrm{OA}$, and many treatment strategies have been developed to protect the cartilage. Although the relationship between cartilage degeneration and subchondral bone destruction is close (Thielen et al., 2019), not all patients with OA exhibit abnormalities in the articular cartilage bone. In addition, in the aging OA phenotype, the imbalance in chondrocyte metabolism occurs before abnormal subchondral bone remodeling (Peffers et al., 2020). In contrast, in the traumainduced OA phenotype, the early micro-injury in subchondral bone is detected first (Barton et al., 2017).

However, increasing evidence demonstrates that maintaining the integrity and remodeling balance of the articular subchondral bone can combat cartilage degeneration to restore homeostasis in joint tissues (Castañeda et al., 2012; Hoshi et al., 2017). Thus, exploring the mechanism underlying subchondral bone remodeling in $\mathrm{OA}$ can provide new insights for developing treatments for early-stage OA. The microstructural changes in articular subchondral bone in $\mathrm{OA}$ include the formation of subchondral bone cysts, bone marrow edema-like lesions, and osteophytes caused by early bone loss, late bone sclerosis, and histopathological changes (Li et al., 2013). These changes are caused by chondrocytes, osteoblasts, osteoclasts, endothelial cells, and the subchondral bone microenvironment (Henrotin et al., 2012).

Osteoblasts differentiate from mesenchymal cells and undergo four stages of maturation, namely, preosteoblasts, osteoblasts, bone-lining cells, and bone cells (Clarke, 2008; Katsimbri, 2017). The phenotype and activity of OA osteoblasts in subchondral bone are altered. For example, the levels of OCN, RANKL, (Kwan Tat et al., 2008) insulin-like growth factor 1, (Hilal et al., 1998) transforming growth factor $\beta 1$, (Abed et al., 2017) vascular endothelial growth factor (Corrado et al., 2013), and alkaline phosphatase activity are elevated in OA, which subsequently lead to osteoclastogenesis, sclerosis (Wang et al., 2013) and angiogenesis. Osteoclasts are multinucleated cells derived from bone marrow myeloid progenitor cells and are mainly responsible for bone resorption and formation (Teitelbaum, 2000; Katsimbri, 2017). During osteoclast formation, progenitor cells are recruited to specific parts of the bone surface to differentiate into osteoclasts (monocytes) and fuse, thereby forming multinucleated mature osteoclasts. Mature osteoclasts adhere to the old bone area and release hydrogen ions and catalytic enzymes to dissolve the bone.

\section{Mesenchymal Stem Cells}

Mesenchymal stem cells (MSCs) are multipotent progenitor cells that originate from the mesoderm. MSCs by default can't regenerate bona fide articular hyaline cartilage (it's primarily fibrous cartilage and hypertrophy) (Buhrmann et al., 2010). However, MSCs can contribute to cartilage and bone repair, and their function in immune regulation and organ regeneration has been extensively studied (Levy et al., 2020). MSCs can be roughly divided into three types: embryonic stem cells, pluripotent stem cells, and adult stem cells (Vizoso et al., 2017).

Pluripotent stem cells are found in bone-related tissues, such as the bone marrow, synovium, infrapatellar fat pad, and adipose tissues (Pers et al., 2015). Bone marrow-derived MSCs (BMSCs) are the most well-characterized pluripotent stem cells. As early as 2002, stem cell therapy based on the in vitro expansion of autologous BMSCs has been used to treat OA. Although there was no significant difference in clinical results, improvement in symptoms was observed from arthroscopic and histological findings (Wakitani et al., 2002). In vitro, synovial MSCs (SMSCs) exhibit a particularly high capacity for cartilage differentiation (Shirasawa et al., 2006; Kurth et al., 2007). Studies have shown that SMSCs from OA patients can repair cartilage through allogenic tissue-engineered constructs in both in vitro and in vivo models. In experimental animal models, the injection of SMSCs into the joint cavity achieved a similar effect (Kondo et al., 2019; Enomoto et al., 2020; Dragoo et al., 2012). Infrapatellar fat pad (IPFP), a column of fat tissue located behind the patella, and synovium are involved in the occurrence and development of intra-articular diseases, such as OA (Favero et al., 2017). As the MSCs/stromal cells derived from IPFP (Kouroupis et al., 2019) are similar to SMSCs, (To et al., 2019) IPFP MSCs are speculated to have the ability for tissue repair (Galipeau et al., 2016), indicating IPFP as a potential target for joint diseases (Attur et al., 2010). Its potential in chondrogenic, osteogenic, and adipogenic lineages has been reported (Sun et al., 2018). In vitro, the chondrogenic differentiation ability of IPFP MSCs was greater than that of BMSCs, adipose tissue MSC (AMSCs), and UC-MSCs (Ding et al., 2015). AMSCs can promote cartilage regeneration and regulate inflammation. Because they are versatile and readily available, AMSCs are an excellent source of cells for OA treatment (Kim and Koh, 2018; 
Lee et al., 2019). However, the mechanism by which AMSCs induce cartilage regeneration remains unclear. Current evidence indicates that AMSCs regulate the local microenvironment through paracrine nutritional factors, thereby making it more favorable for regeneration and repair and subsequently delaying cartilage degradation and improving joint function (Damia et al., 2018). Previously, a small number of MSC-like progenitor cellschondrogenic stem cells/progenitor cells (CSPCs) were detected in cartilage tissues (Alsalameh et al., 2004; Fickert et al., 2004; Koelling et al., 2009). Because they share similar properties with BMSCs, CSPCs are speculated to be involved in cartilage regeneration. CSPC migration occurs upon cartilage damage, and their proliferation and immune regulation capabilities are enhanced (Seol et al., 2012; Riegger et al., 2018).

\section{Induced Pluripotent Stem Cells and Tissue-Engineered Cells}

Induced pluripotent stem cells (iPSCs) can provide an unlimited cell source for tissue engineering and are an attractive substitute for primary cells. iPSCs are characterized by a high degree of plasticity and promising differentiation potential and have promising potential in cell therapy. Patient-specific iPSCs can be engineered to minimize the autoimmune response, making them an almost ideal cell source for cell-based therapy. Studies on cartilage tissue engineering of iPSCs have demonstrated their utility for functional cartilage repair and as models for studying cartilage pathology (Diekman et al., 2012; Willard et al., 2014). iPSCs provide a platform for identifying candidate, patientspecific OA therapeutic agents (Lietman, 2016). For example, iPSCs reprogrammed from somatic cells (Takahashi et al., 2007; $\mathrm{Yu}$ et al., 2007) could generate endless OA patient-specific stem cells for drug research. Moreover, iPSCs isolated from the tissues of $\mathrm{OA}$ patients could differentiate into the cartilage, which provides opportunities for cartilage tissue research (Wei et al., 2012; Lach et al., 2014; Nam et al., 2018). However, there are no clinical studies published about cartilage cell therapy using iPSCs. iPSCs not only have excellent proliferation and differentiation capabilities similar to other stem cells but also do not cause immune rejection and ethical issues (Moradi et al., 2019). Therefore, more studies are required to improve the future applications of iPSC-derived chondrocytes in OA replacement therapy.

\section{VARIOUS MECHANISMS OF CELLULAR CROSSTALK IN OA PATHOGENESIS}

\section{Microenvironment, Paracrine Signals, and Co-culture Method \\ Cartilage and Subchondral Bone}

In joints with OA, some blood vessels of the subchondral bone can penetrate calcified cartilage and even invade non-calcified cartilage (Chen et al., 2015). Osteoclast precursors invade the area of hypertrophic cartilage and interact with its cells to reshape the cartilage matrix and form an ossification center (Tonna et al., 2016). In addition, mature osteoclasts could regulate nearby chondrocytes, which destroys the connection between the bone and cartilage and degrade articular cartilage via cysteine proteases and matrix metalloproteinases (Löfvall et al., 2018). Osteoclasts could aggravate cartilage damage by regulating chondrocytes. The expression level of TGF- $\beta 1$ in osteoclasts was upregulated in a time-dependent and dose-dependent manner under mechanical stimulation. Upon co-culture with osteoclasts, chondrocytes showed aggravated apoptosis. The injection of TGF- $\beta 1 \mathrm{R}$ inhibitor into the abdominal cavity of rats with OA effectively reduced chondrocyte apoptosis and cartilage degradation (Zhang R. K. et al., 2018). TGF- $\beta 1$ was not derived from osteoclastic bone resorption but was transported from the subchondral bone to the cartilage layer via diffusion or blood circulation to adversely affect chondrocytes. The cartilage can also obtain calcium-phosphate complexes from subchondral bone via p38, ERK1/2, nuclear factor- kappa B (NF- $\mathrm{B})$, signal transducer, and activator of transcription 3 (STAT3) to increase the production of MMP-13 in chondrocytes (Jung et al., 2018).

Chondrocytes can also promote the loss of subchondral bone by regulating osteoclasts. Abnormal mechanical stress could induce IL-1 $\beta$ production in primary chondrocytes (Fujisawa et al., 1999). IL-1 $\beta$ increased the expression of receptor activator of NF- $\kappa \mathrm{B}$ ligand (RANKL) through osteoblasts, thereby indirectly inducing the generation and maturation of osteoclasts (Cao et al., 2016). In an OA model induced by destabilization of the medial meniscus (DMM), chondrocytes produced large amounts of TNF- $\alpha$ and IL-6 (Pearson et al., 2017). TNF- $\alpha$ activates NF- $\kappa \mathrm{B}$ and $\mathrm{c}$-Jun $\mathrm{NH} 2$-terminal protein kinase (JNK) in a RANKL-independent manner to directly induce osteoclast differentiation (Kobayashi et al., 2000) and indirectly induce its production (Tanaka et al., 2005). In an in vivo OA model, the expression of high-mobility group box 1 (HMGB1) was detected in chondrocytes (Aulin et al., 2020). As demonstrated by the bone development of $H M G B 1^{-/-}$hypertrophic chondrocytes in the mouse growth plate, endochondral bone formation was disrupted due to the delayed invasion of osteoclast precursors into the primary ossification center (Taniguchi et al., 2007). In addition, senescent chondrocytes and hypertrophic chondrocytes produced pro-inflammatory mediators, catabolic enzymes, and chemokines, collectively known as the senescence-associated secretory phenotype (SASP), (Rim et al., 2020) to affect subchondral osteoclast lineage cells.

\section{Infrapatellar Fat Pad and Synovium}

In OA, IPFP and nearby synovium also experience inflammatory infiltration and hyperplasia (Favero et al., 2017). IPFP releases IL-6, IL-8, prostaglandin F2a (PGF2 $\alpha$ ), and TNF $\alpha$, which subsequently causes fibrosis of the synovium (BastiaansenJenniskens et al., 2013; Eymard et al., 2014). In vitro, fibroblastlike synovial cells of OA patients pre-treated with TGF $\beta$ or PGF2 $\alpha$ inhibitors were cultured in the conditioned medium derived from IPFP tissues for 4 days and exhibited different migration and proliferation abilities. Hyperplasia and fibrosis also occurred (Bastiaansen-Jenniskens et al., 2013). In addition, adipocytes derived from IPFP could regulate macrophages and $\mathrm{CD} 4+\mathrm{T}$ cells infiltrating into the synovium by secreting lipids (Ioan-Facsinay et al., 2013; Klein-Wieringa et al., 2013). 
The free fatty acids found in the conditioned medium derived from IPFP adipocytes improved the proliferation of CD4 $+\mathrm{T}$ cells and their ability to produce IFN- $\gamma$. These free fatty acids could also reduce the secretion of IL-12p40 cytokine by macrophages (Klein-Wieringa et al., 2013); IL-12p40 is a chemoattractant that can induce macrophage inflammation and fibrosis (Cooper and Khader, 2007).

Macrophages have been used as potential therapeutic targets, and their pharmacological depletion and phenotypic changes in IPFP and synovium have been explored (Fernandes et al., 2020; Wu et al., 2020). Macrophages can be induced to polarize back to another anti-inflammatory M2 phenotype. Although there are no reports on the MSC-mediated direct regulation of the phenotype of synovial macrophages in patients with OA, MSCs have been demonstrated to block the activation of M1 macrophages and promote the polarization of M2 macrophages to inhibit inflammation in vitro (Harrell et al., 2019). MSCs first migrate to the tissue injury site and promote the polarization of M2 macrophages by secreting a large number of cytokines, chemokines, and growth factors (Fernandes et al., 2020), thereby enhancing the repair of damaged tissues. The intra-articular injection of MSCs could downregulate the level of iNOS in macrophages and reduce the formation of M1 macrophages (Hamilton et al., 2019). Interestingly, in OA joints, M1 macrophages subsequently inhibited the proliferation and viability of MSCs, enhanced the immune response, and ultimately aggravated cartilage degradation.

Infrapatellar fat pad can also interact with the cartilage. The main limitation of MSC-based cartilage constructs is the induction of a hypertrophic phenotype during in vivo differentiation, which leads to endochondral ossification (Scotti et al., 2013; Correa et al., 2015; Feng et al., 2018). However, 8 weeks after implementing hybrid structures in which IPFP MSCs and articular chondrocytes were co-cultured in nude mice, cartilage mineralization was reduced, and the phenotype was stable (Mesallati et al., 2015). IPFP MSCs could accumulate a large amount of sGAG on articular cartilage agarose gels, thereby improving the mechanical properties of tissueengineered articular cartilage constructs (Mesallati et al., 2017).

\section{Cell Co-culture}

Co-culture of chondrocytes and synovial cells has been used in cartilage research as an in vitro model for OA. Cocultured chondrocytes and synovial cells stimulated by proinflammatory cytokines interact via calcium signaling and paracrine pathway to maintain the homeostasis of chondrocytes. This co-culture method allows for the accurate evaluation of the role of anti-inflammatory or chondroprotective molecules in the articular cartilage (Beekhuizen et al., 2011). The co-culture of osteoblasts and chondrocytes can also be used to study the role of chondroprotection (via delaying the onset of cartilage degradation) in bone remodeling. Paracrine signals are also used to maintain the physiological state and phenotype of the cells (Thysen et al., 2015). MSCs promote specific dedifferentiation due to their pluripotency. When co-cultured with other cells, different cell pathways can be analyzed together with articular chondrocytes with cell secretion markers (Hendriks et al., 2007).
However, the co-cultivation method also has certain limitations, including restricted growth of cells and high cost.

\section{Intercellular Signaling by EVs}

Extracellular vesicles (EVs) are nano-sized communication messengers secreted by cells that transmit biological signals between cells and are mainly divided into three categories, namely, exosomes, microvesicles, and apoptotic bodies. EVs have received increasing research attention in the field of regenerative medicine. Increasing studies have demonstrated potential value for alleviating inflammation and promoting tissue repair and regeneration (Bjørge et al., 2017).

Extracellular vesicles are small double lipid membrane vesicles with a diameter of approximately 30-2000 nm that can carry various biologically active molecules, such as RNA subtypes (mRNA, microRNA, and lncRNA), DNA fragments, lipids, proteins, and enzymes (Lamichhane et al., 2015). Most cells, such as those in connective tissues and MSCs, can produce and secrete EVs into various biological fluids, such as the blood and synovial fluid (Rani et al., 2015). Once released, EVs can take effect immediately or be transported to a distant place. EVs communicate with the recipient cell by producing the same effect as their donor cell.

Exosomes are small vesicles with a diameter of approximately 30-150 nm derived from the endosomal compartment. They are the most widely studied type of EVs (Théry et al., 2002). Endosomal membrane invaginates to form multivesicular bodies containing intraluminal vesicles. When multivesicular bodies fuse with the plasma membrane, intraluminal vesicles are secreted as exosomes into the extracellular space. Microvesicles are slightly larger than exosomes, with a diameter ranging between 50 and $1000 \mathrm{~nm}$ (De Jong et al., 2014). Because microvesicles are directly shed from the plasma membrane, their markers on the membrane surface are also the same as the donor cells. Similar to exosomes, microvesicles can transport biologically active molecules to recipient cells. Apoptotic bodies are the largest type of EVs, with a diameter of $\geq 1000 \mathrm{~nm}$, and are formed in the late-stage of apoptosis (De Jong et al., 2014). Apoptotic bodies may function as biomarkers. However, at present, the connection between regenerative medicine and cells remains unclear.

Extracellular vesicles serve as mediators of intercellular communication (Raposo and Stoorvogel, 2013; De Jong et al., 2014). EVs interact with the surface receptors of recipient cells through their transmembrane proteins to activate downstream intracellular signaling pathways. EVs can also be directly endocytosed by recipient cells to release their contents (Jaiswal et al., 2014). The type of donor cells and the environment in which they are located, such as being in a state of stress, can affect EVs' function and contents (de Jong et al., 2012). The biological functions of EVs and their biogenesis require more studies. Increasing studies in regenerative medicine have focused on the production of protective and pro-regenerative EVs, particularly in cartilage repair.

Studies have revealed the role of EVs in OA pathogenesis, inflammation, and cartilage regeneration and have demonstrated their potential implications for joint disease therapy 
(Zhang S. et al., 2018). EVs derived from different types of joint cells participate in maintaining joint homeostasis and can initiate and promote the progression of OA (Murphy et al., 2018). Macrophages and leukocytes infiltrating the synovium could interact with fibroblast-like synovial cells through EVs (Malda et al., 2016). The activated fibroblast-like synovial cells then transmit inflammatory signals, such as cytokines and enzymes, to the macrophages and leukocytes, thus forming a feedback loop that further aggravates OA. These EVs could also cause the degradation of the extracellular matrix and result in changes in the subchondral bone. In an in vitro OA model, EVs enhanced cartilage anabolism and relieved inflammation (Wang et al., 2017), thereby delaying cartilage degradation and the progression of OA (Zhu et al., 2017). In addition, EVs protected chondrocytes (Headland et al., 2015) and regulated the physiological activities of various types of immune cells (Lo Sicco et al., 2017), indicating their anti-inflammatory effects. The induction of a regenerative immune phenotype and enhanced metabolic level of chondrocytes promote the formation of type II collagen-rich cartilage that can repair cartilage defects in rat and rabbit OA models (Zhang et al., 2016; Zhang S. et al., 2018). Hydrogel encapsulation delivers EVs at more accurate positions and higher doses, thereby significantly enhancing repair ability (Liu et al., 2017).

Recently, the application of stem cell-derived EVs in the treatment of joint damage and $\mathrm{OA}$ has received increasing attention. They have been derived from the bone marrow (Cosenza et al., 2018; Vonk et al., 2018), adipose tissue (Lo Sicco et al., 2017), synovium (Zhu et al., 2017), or pluripotent cells, including embryonic stem cells (Zhang et al., 2016; Zhang S. et al., 2018) and iPSCs (Liu et al., 2017; Zhu et al., 2017). Exosomes are the main research target in EVs. The difference between exosomes and microvesicles derived from the same cell has been investigated (Cosenza et al., 2017, 2018). Moreover, EVs containing both exosomes and microvesicles have been analyzed (Headland et al., 2015; Lo Sicco et al., 2017). In addition to sharing similar biological functions with stem cells, stem cell-derived EVs offer significant advantages due to their small size and low immunogenicity. Issues associated with direct cell injection can also be avoided. EVs do not pose the risk of antigen presentation due to differentiation into specific cell types such as MSCs, which allows them to be used in allogeneic therapy. In addition, the biologically active cargoes inside MSC-derived EVs are more stable, and problems such as senescence following expansion or cartilage calcification following induction are eliminated.

\section{Exosomes Derived From Joint Tissues}

Exosomes maintain homeostasis (Gao et al., 2018) and facilitate cell-to-cell communication in diseases (Li Z. et al., 2018). The source and content of exosomes determine their functions and biological characteristics. Exosomes secreted by therapeutic cells help treat diseases, while those released by cells in the pathological microenvironment accelerate the disease process. In this section, we discuss the various kinds of exosomes derived from joint tissues and their biological effects in OA.

\section{Cartilage-Derived Exosomes}

Osteoarthritis chondrocytes release extracellular articular cartilage matrix vesicles with a diameter of $100 \mathrm{~nm}$ and participate in the pathologic mineralization of articular cartilage (Anderson, 2003; Jubeck et al., 2008). Exosomes and articular cartilage matrix vesicles share similar features, including size, morphology, and lipid and protein content (Shapiro et al., 2015), suggesting that they exhibit homologous functions with respect to cell communication (Ni et al., 2019). Exosomes derived from normal primary chondrocytes (D0 exosomes) could restore mitochondrial function and enhance immune infiltration by increasing the ratio of M2/M1 macrophages. The intra-articular injection of D0 exosomes effectively suppressed the occurrence and development of OA (Zheng et al., 2019). OA chondrocyte-derived exosomes stimulated the activation of inflammasomes in macrophages and released mature IL- $1 \beta$ via the miR-449a-5p/ATG4B/autophagy pathway, thereby inducing synovitis and exacerbating $\mathrm{OA}$ (Ni et al., 2019). In addition, exosomes derived from chondrocytes could achieve efficient ectopic chondrogenesis of cartilage progenitor cell (CPC) constructs, representing a novel cell-free therapeutic approach for cartilage regeneration (Chen et al., 2018).

\section{Synovial-Derived Exosomes}

Microvesicles derived from neutrophils have been shown to penetrate cartilage, implying that EVs from the synovium could meditate the crosstalk between the synovium and cartilage (Headland et al., 2015). Kato et al. employed IL-1 $\beta$ to stimulate normal SFB, isolated the secreted exosomes, and co-cultured them with chondrocytes. They found that the expression of catabolic-related genes, such as MMP13 and ADAMTS-5, significantly increased, whereas that of anabolic-related genes, such as COL $2 A 1$ and $A C A N$, significantly decreased. In addition, these exosomes promoted the production of proteoglycan from cartilage explants (Kato et al., 2014). These findings indicate that synovial-derived exosomes could induce OA-like phenotype both in vivo and in vitro.

Exosomes derived from other synovial cells, such as macrophages, have also been studied. For instance, the effects of salazosulfapyridine and methotrexate on the proteome of exosomes produced by a human synovial sarcoma cell line (SW982) have been investigated. Tsuno et al. observed that these anti-rheumatic drugs altered the protein profiles of SW982derived exosomes and inhibited the effect of IL-1 $\beta$ on the exosomal proteome (Tsuno et al., 2016).

\section{Subchondral Bone-Derived Exosomes}

Exosomes derived from cells in the subchondral bone, including osteoblasts, osteoclasts, and bone marrow mesenchymal stem cells, regulate the microenvironment of subchondral bone (Li et al., 2016; Sun W. et al., 2016). Osteoblasts in the subchondral bone of patients with different degrees of OA secreted exosomes positive for HSP70, CD9, and flotillin-1 (exosomal markers) and a diameter ranging between 30-150 nm. In addition, these exosomes contained a large number of miRNAs, such as miR-135a-3p, miR-210-5p, miR-885-3p, and 
miR-1225-5p. Exosomes derived from cells in other subchondral bones also have diagnostic value.

\section{Therapeutic Potential of Stem Cell-Derived Exosomes in OA}

Stem cells, such as BMSCs and AMSCs, promote cartilage regeneration and have been used in clinical trials for $\mathrm{OA}$ treatment (Lee and Wang, 2017; De Bari and Roelofs, 2018). The safety and feasibility of intra-articular injection of MSC have been confirmed (Di Matteo et al., 2019), which can partially relieve knee joint pain (Yokota et al., 2019) and improve the knee society clinical rating system (KSS) and the outcome score of OA (Jo et al., 2017; Kim et al., 2020). Stem cells exert therapeutic effects mainly through their paracrine functions, such as the secretion of EVs (Phinney and Pittenger, 2017). In this section, we summarize the recent studies on exosomes derived from different types of stem cells, focusing on their roles in the occurrence and development of OA and their therapeutic potential.

\section{BMSC-derived exosomes}

Exosomes derived from BMSCs (BMSC-Exos) can promote the regeneration and repair of damaged cartilage and subchondral bone (Mianehsaz et al., 2019; Asghar et al., 2020). The exosomes and microvesicles from TGF $\beta 3$-pre-treated BMSCs increased the expression of anabolic markers and decreased the levels of catabolic marker genes in osteoarthritic chondrocytes. In addition, these BMSC-derived exosomes could prevent osteoarthritic chondrocytes from undergoing apoptosis (Cosenza et al., 2017). BMSC-Exos could be taken up by chondrocytes to abolish damage to the mitochondrial membrane potential and IL-1 $\beta$-induced apoptosis (Qi et al., 2019). BMSC-Exos could also affect the phenotype of other cells in OA joints by inhibiting the activity of osteoclasts in subchondral bone and activating macrophages in the synovium (Li et al., 2020), and suppressing the proliferative activity of SFB pre-treated with IL- $1 \beta$ and increasing its apoptosis. In an in vivo OA model, the injection of BMSC-Exos into the joint cavity abrogated the damage and degradation of cartilage and subchondral bone and decreased synovial tissue proliferation and inflammatory cell infiltration, thereby alleviating the symptoms of OA (Jin et al., 2020).

Genetic modification or drug intervention can influence the effect of exosomes on recipient cells by regulating the secretion and contents of exosomes (Ma et al., 2017; Liu and Su, 2019). Exosomes secreted by BMSCs overexpressing miR-92a-3p were collected and applied to chondrocytes. The expression levels of SOX9, COL2A1, and aggrecan increased, whereas those of RUNX2 and MMP13 decreased, indicating that modified BMSC-Exos greatly enhance cartilage repair (Mao et al., 2018). In addition, in vivo, and in vitro experiments showed that kartogenin-pre-treated BMSC-Exos more significantly enhanced cartilage formation and damage repair than normal BMSC-Exos (Liu et al., 2020).

\section{BMSC-derived exosomes}

In vitro synovial mesenchymal stem cells (SMSCs) possess an exceptionally high capacity for cartilage differentiation (Kurth et al., 2007). One study using allogenic tissue-engineered constructs reported that SMSCs from OA patients effectively enhanced cartilage repair (Koizumi et al., 2016). In animal OA models, the injection of SMSC into the joint cavity inhibited the occurrence and development of OA (Ozeki et al., 2016; Kondo et al., 2019). Exosomes derived from SMSC (SMSC-Exos) could not only induce the proliferation and migration of chondrocytes but also reduce the secretion of ECM. Interestingly, SMSCExos transfected with miR-140-5p blocked damage to ECM, effectively reduced joint damage, lowered the OARSI score, and delayed the occurrence and development of OA (Tao et al., 2017). In addition to maintaining cartilage homeostasis, SMSCExos can regulate bone remodeling (including subchondral bone changes and osteophyte formation) by reducing glucocorticoidinduced fat cell accumulation, trabecular bone loss, and bone marrow necrosis. In addition, SMSC-Exos could partially reverse proliferation arrest and glucocorticoid-induced apoptosis of BMSCs (Guo et al., 2016).

\section{IPFP MSC-derived exosomes}

Infrapatellar fat pad plays a key role in knee joint function and pathology. IPFP-derived MSCs (IPFP MSCs) have been suggested as promising cell sources for OA treatment owing to their potent capability for cartilage regeneration (Buckley et al., 2010; Koh and Choi, 2012). In a DMM-induced OA mouse model, exosomes derived from IPFP MSCs effectively reduced cartilage damage and improved abnormal gait. RNA sequencing analysis of the exosomes revealed high miR-100$5 p$ levels, indicating that exosomal IPFP MSCs may inhibit the mTOR pathway via miR-100-5p to regulate chondrocyte phenotype (Wu et al., 2019). The physiological and pathological effects of other exosomes in the IPFP, such as those secreted by adipocytes, also have research value.

\section{AMSC-derived exosomes}

Although the mechanism by which AMSCs induce cartilage regeneration is unclear, mounting evidence suggests that AMSCs regulate the cartilage microenvironment by secreting paracrine growth factors (Damia et al., 2018). EVs, including exosomes and microvesicles, mainly mediate the paracrine effects of osteoblasts in OA. AMSC-derived exosomes (AMSCExos) reduced the accumulation of senescence-associated $\beta$ galactosidase and $\gamma \mathrm{H} 2 \mathrm{AX}$ foci in osteoblasts pre-treated with IL-1 $\beta$, decreased the levels of PGE2 and IL- 6 , increased that of IL-10, and downregulated the mitochondrial membrane potential (Tofiño-Vian et al., 2017). In addition, AMSC-Exos could inhibit the production of pro-inflammatory mediators, such as TNF- $\alpha$ and NO, and suppress the activity of MMP while enhancing that of anti-inflammatory cytokines, such as IL-10 and type II collagen. These findings indicate the antiinflammatory and chondroprotective effects of AMSC-Exos (Tofiño-Vian et al., 2018). AMSC-derived EVs (86.46 nm in diameter) promoted the proliferation and migration of $\mathrm{OA}$ chondrocytes and maintained the metabolic balance of ECM. In monosodium iodoacetate (MIA) rat and DMM mouse models, the injection of AMSC-derived EVs into the joint cavity effectively delayed OA progression and showed protective effects against cartilage degeneration (Woo et al., 2020). ADSCs-Exos could downregulate the expression of pro-inflammatory genes 
in SFB and increase anti-inflammatory cytokines, promoting the proliferation and cartilage formation of periosteal cells via miR145 and miR-221 (Zhao C. et al., 2020). Overall, AMSC-Exos has great therapeutic potential for the treatment of OA.

\section{Embryonic mesenchymal stem cell (EMSC)-derived exosomes} Embryonic mesenchymal stem cells are another potential candidate for cartilage regeneration and OA treatment (Mamidi et al., 2016; Gibson et al., 2017). Recently, exosomes derived from embryonic MSCs (EMSC-Exos) have been reported to regulate the phenotype of chondrocytes and delayed OA progression (Zhang S. et al., 2018; Zhang et al., 2019). After successfully isolating and identifying EMSC-Exos, Zhang et al. (2016) injected them into osteochondral defects in rats. After 6 weeks, cartilage and subchondral bone damage were largely reversed, and complete recovery was achieved at 12 weeks. Similarly, exosomes derived from the E1-MYC 16.3 human embryonic stem cell line reduced the production of M1 macrophages and pro-inflammatory cytokines and increased the infiltration of M2 macrophages. In addition, this study observed that EMSC-Exos could be endocytosed by chondrocytes to regulate their chondrocyte proliferation, migration, and matrix synthesis (Zhang S. et al., 2018). In an OA model of the temporomandibular joint in rats, EMSC-Exos reduced inflammation, alleviated early pain, and promoted cartilage repair and subchondral bone healing. The activation of AKT, ERK, and AMPK pathways can also reverse IL-1 $\beta$-induced production of MMP13 and NO and inhibit sGAG synthesis (Zhang et al., 2019). TGF- $\beta 1$ increases miR-135b levels in EMSCExos, thereby reducing the expression of $\mathrm{Sp} 1$ to promote the proliferation of chondrocytes and accelerate cartilage repair (Wang et al., 2018).

\section{Exosomes derived from other stem cells}

Exosomes derived from amniotic fluid stem cells (AFSC-Exos) in an MIA-induced OA model improved the pain tolerance, induced the restoration of regular hyaline cartilage, and inhibited the polarization of M1 macrophages, suggesting that AFSC-Exos can regulate inflammation (Zavatti et al., 2020). Meanwhile, those derived from umbilical mesenchymal stem cells (UMSCExos) induced chondroprotective effects, including increased proliferation and migration of chondrocytes, increased ECM synthesis, and reduced cell apoptosis. UMSC-Exos produced from $3 \mathrm{D}$ culture enhanced cartilage repair compared to those from 2D culture (Yan and Wu, 2020). Exosomes secreted by MSCs derived from pluripotent stem cells (with a diameter of approximately 50-150 nm; iMSC-Exos) significantly promoted the proliferation and migration of chondrocytes and had improved efficacy for OA treatment compared with SMSC-Exos (Zhu et al., 2017).

However, how the exosomes in joint tissues and cells participate in OA initiation remains unclear. In addition to their positive therapeutic effects, exosomes in the OA microenvironment may exert unwanted effects, including promotion of inflammation and inhibition of cartilage repair. Therefore, it is also imperative to explore the mechanism of "negative" exosomes in OA. A recent study on exosomes in the plasma and synovial fluid reported their diagnostic value in patients with OA (Zhao and $\mathrm{Xu}, 2018$ ). However, the exosomes shared by these two tissues could not distinguish between earlystage and late-stage OA. Notably, the expression level of exosomal lncRNA PCGEM1 in synovial fluid was significantly higher in patients with advanced OA than in those with early OA and was higher in early OA, indicating that exosomal lncRNA PCGEM1 from synovial fluid may be a powerful indicator for distinguishing early-stage and late-stage OA. lncRNA PCGEM1 acts as sponge lncRNA targeting miR-770 and promotes the proliferation of synovial cells (Kang et al., 2016). In general, the clinical utility of exosomes as diagnostic biomarkers for OA diagnosis is in its preliminary stages.

\section{Cell Tissue Engineering}

Recent attempts to differentiate iPSCs derived from OA patients into chondrocytes have been conducted. Generally, the addition of growth factors, such as TGF- $\beta$, FGF-2, BMP, and WNT3A, and paracrine factors, such as Ihh and Runx, to the culture medium is necessary to drive iPSCs to the chondrogenic lineage (Yamashita et al., 2018; Zhao Y. et al., 2020). Currently, four main methods are available: (1) transformation of iPSCs into MSC-like cells and their differentiation into chondrocytes (Nejadnik et al., 2015); (2) co-culture of MSCs derived from iPSCs with primary chondrocytes (Qu et al., 2013); (3) formation of embryoid bodies (EBs) (Nakagawa et al., 2009); (4) cultivation of iPSCs in a medium that mimics the physiological environment during development (Diekman et al., 2012). Studies aiming to form iPSCs through EB and co-culture them with chondrocytes have been conducted (Wei et al., 2012). First, the chondrocytes of OA patients were reprogrammed into OA-iPSCs by lentivirus induction. After the formation of EB, they were continually cultured in the chondrogenic medium for 14 days. Subsequently, the iPSCs were transfected with a lentivirus carrying TGF-1 $\beta$ and inoculated on alginate matrix-coated dishes. After culturing for another 14 days, TGF-1 $\beta /$ iPSCs were subcutaneously injected into the back of mice. Ectopic cartilage tissue was observed at 6 weeks after transplantation.

Engineered cartilage tissue from chondrocytes, when transiently transfected circuits activate the PTGS2 gene, immunomodulatory IL-4 is produced, thereby representing a new immunomodulatory method (Nims et al., 2021). Autologous articular chondrocytes are an established cell-based tissue engineering strategy for treating knee cartilage or osteochondral defects (Brittberg et al., 1994). Most of the current scaffolds or biomaterials contain MSCs that can undergo chondrogenic differentiation and are used as clinically relevant chondrogenic implants to repair cartilage defects. However, after their implantation in the body, differentiated chondrocytes showed a hypertrophic phenotype (collagen X, MMP13) and induced ectopic bone formation (Pelttari et al., 2006). Therefore, the production of articular chondrocytes with stable, extracellular matrix and phenotype is the main goal of in vitro cartilage tissue engineering.

Macrophages are among the main types of cells that affect joint homeostasis and have been applied for the development of related cell engineering technologies. In addition 
to using biomaterial scaffolds to regulate macrophage-induced inflammation, macrophages themselves have been utilized for drug delivery or treatment. The intrinsic homing ability of macrophages allows their migration to the site of inflammation or injury in OA. Using this feature, autologous M1 macrophages are used to deliver nanoparticle-encapsulated drugs to induce transient phagosome maturation arrest (Visser et al., 2019). The regular use of clustered interspaced short palindromic repeats (CRISPR)-Cas9 genome editing to create a cell-autonomous system ("SMART" cell), which is derived from mouse induced pluripotent stem cells (miPSCs), has also been attempted. Chondrocytes can automatically regulate inflammation in both in vivo and in vitro OA models. When "SMART" chondrocytes receive specific targeting signals (such as inflammatory cytokines IL-1 or TNF- $\alpha$ ), they released corresponding biological drugs, such as IL-1Ra and or soluble TNFR1, to relieve inflammation (Brunger et al., 2017; Pferdehirt et al., 2019). In the same way, the design of self-regulating "SMART" macrophages enables these cells to not only automatically home to the inflammation/injury site but also to have cytokine-activated feedback-controlled capabilities. Thus, targeted drugs can be effectively delivered to treat joint diseases (Adkar et al., 2017). Overall, cell tissue engineering is a powerful tool to develop new modalities for OA treatment.

\section{CONCLUSION AND PROSPECTS}

This review discusses the functions of different types of cells in the joints and their roles in $\mathrm{OA}$, the interaction among various joint cells and tissues, and the latest cell tissue engineering techniques.
Our article provides a comprehensive summary of the complex mechanisms underlying the occurrence and development of OA and potential targets of future therapies for OA (Figure 1). Among the various cell types, MSCs and their secreted products (EVs) are the focus of future research (Figure 2).

Increasing studies have demonstrated the clinical applications of MSCs (Mendicino et al., 2014), such as their role in promoting cartilage repair and delaying the progression of OA (Lee and Wang, 2017). In phase I and II clinical trials, the injection of MSCs into the joint cavity is reportedly reliable and safe. A 5-year follow-up survey demonstrated the efficacy of MSCs in improving cartilage quality and joint function (Yubo et al., 2017; McIntyre et al., 2018). However, the use of MSCs for OA treatment has certain limitations. For example, cell survival and long-term cell behavior after injection are difficult to predict, and the maintenance of cell banks is also a major challenge (Heldring et al., 2015). The quality of MSCs from different donors varies, particularly from elderly or deceased donors who have reduced proliferation capacity and physiological function. In addition, the in vitro expansion of MSCs causes senescence, proliferation decline, and even dedifferentiation (Siddappa et al., 2007). MSCs are also "environmentally responsive," and changes in the microenvironment can cause drastic changes in cell behavior (Murphy et al., 2013). For example, the stimulation of AMSCs by TNF changed the phenotype and resulted in the secretion of pro-inflammatory proteins, which aggravated the inflammatory response (Lee et al., 2010).

Immunotherapy and nanotechnology can help overcome these limitations. At present, genetic engineering techniques, including the use of viral vectors and CRISPR-Cas9 genome

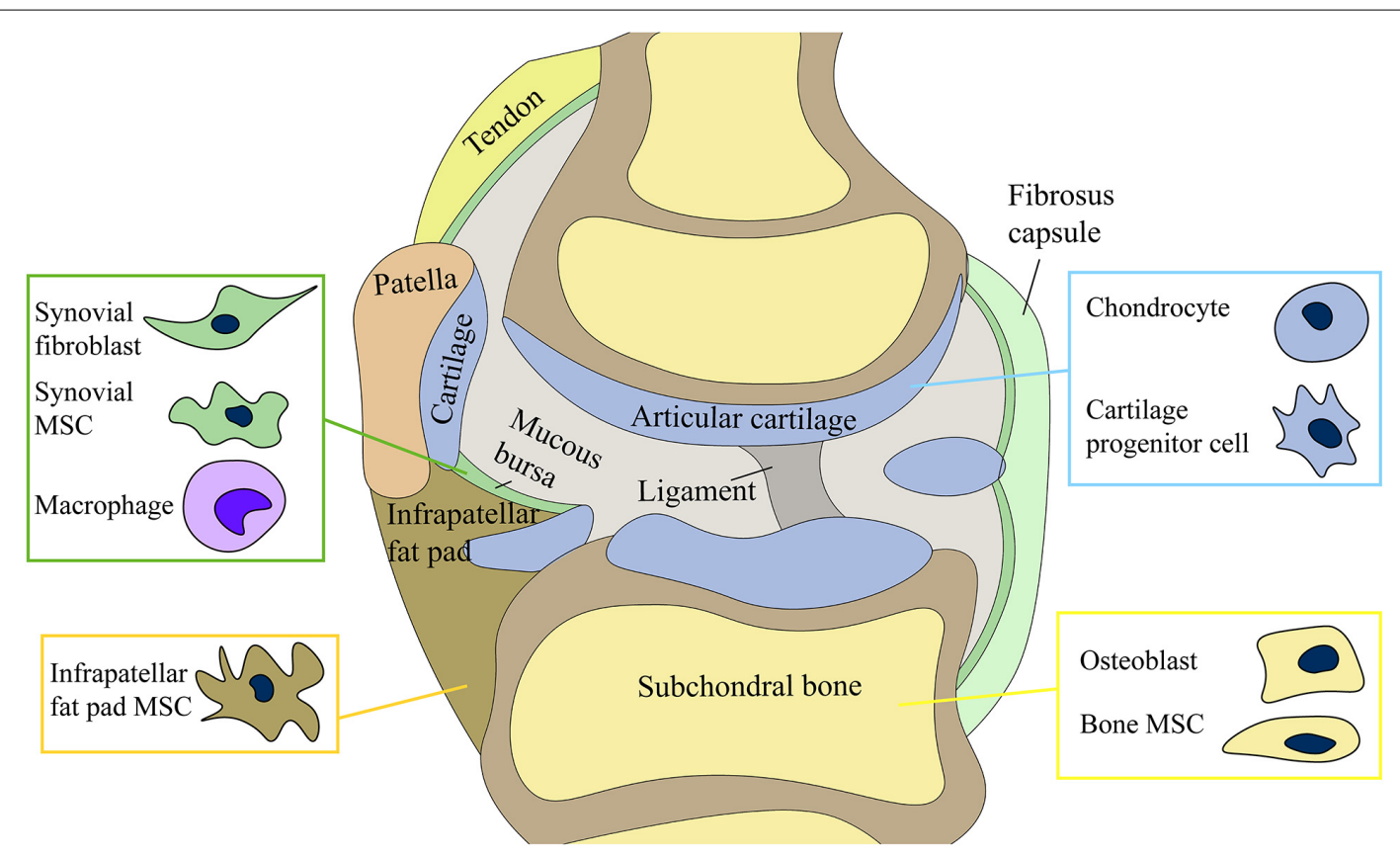

FIGURE 1 | Joint cells) include chondrocytes, osteoblasts of subchondral bone, synovial mesenchymal stem cells (MSCs) and fibroblasts, and infrapatellar fat pad MSCs. The production and release of exosomes by stem cells may be involved in the regulation of joint homeostasis. 


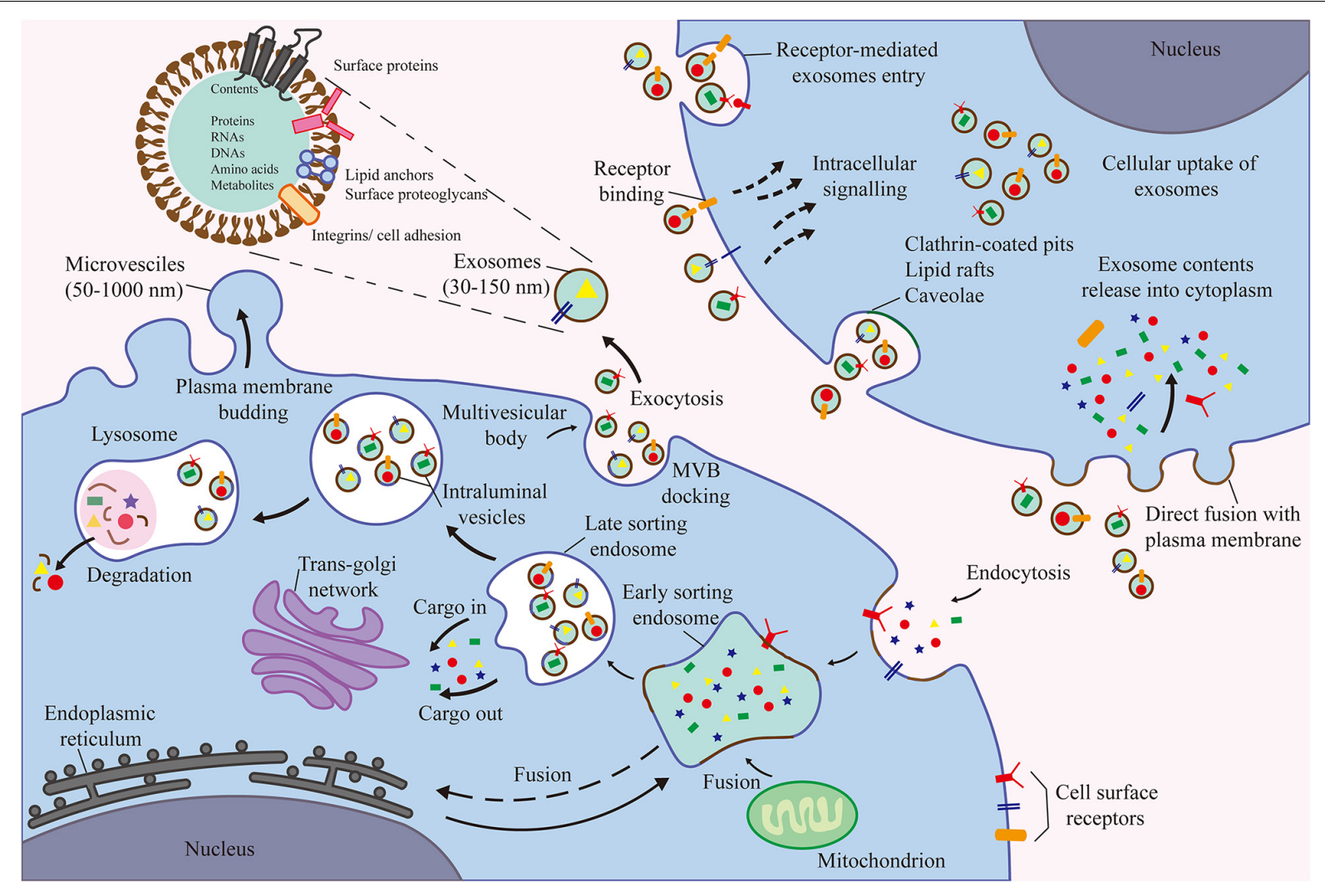

FIGURE 2 | The contents of exosomes are proteins, nucleic acids, amino acids, and metabolites. Extracellular components enter the cell through endocytosis and plasma membrane invagination. Plasma membrane buds are formed on the cavity side and fuse with the components of the endoplasmic reticulum, trans-Golgi network, and mitochondria to form early sorting endosomes. Then, the late sorting endosomes modify the cargo and produce and form various intraluminal vesicles and multivesicular bodies (MVB). Among them, some MVBs degrade after fusion with lysosomes. Other MVB can be transported to the plasma membrane to release intraluminal vesicles as exosomes outside the cell through exocytosis.

editing (Gerace et al., 2017; Pawitan et al., 2020), have been proposed to improve the immune regulation of MSCs. The biological scaffold carrying MSCs expressed IL-1Ra under the influence of exogenous doxycycline, which induces inflammation resistance, contributing to the recovery of degenerative articular cartilage, indicating that cell engineering combined with biological materials can enhance the immunomodulatory ability of MSCs (Glass et al., 2014).

Exploring the function of the products secreted by MSCs, such as EVs, is an alternate direction for OA treatment. The application of stem cell-derived EVs in OA treatment is an emerging field in regenerative medicine. The cargoes delivered by EVs are the same as those of donor cells; however, the former is simpler, more practical, and safer than direct cell transplantation. Exosomes serve as an important mediator of cellular interaction during OA development and have tremendous therapeutic potential. However, this research area has some challenges. At present, there is no direct evidence for the delivery and transfer of endogenous exosomes between cells, which makes identifying the recipient cells of exosomes difficult. The mechanism of exosome production and release in joints also remains unclear, limiting the development of cell therapies that target exosomes. Because cartilage destruction is not severe in early-stage OA, exosomes cannot effectively penetrate the cartilage matrix to interact with cartilage cells. Therefore, current studies on MSCExos cell engineering mainly focus on surface chondrocytes, cartilage matrix, synovial cells, and other joint cells that can easily communicate with exosomes.

Future studies on EVs should explore their cell sources, optimized conditions for the production of EVs, their content and biodistribution in the joints, the type of recipient cells or tissues, and their therapeutic mechanisms in OA. Engineered products based on EVs are expected to further promote the interaction between cells, and the intersection between biology and engineering technology can further optimize the function and production of EVs. Finally, it can represent a relatively complete treatment strategy to reduce the burden of OA patients.

A better understanding of the various interactions among cells and tissues in the joint in OA pathogenesis paves the development of future cell-based therapies for OA treatment.

\section{AUTHOR CONTRIBUTIONS}

ZL conducted the literature review, drafted the manuscript, and prepared the figures. $\mathrm{ZH}$ and $\mathrm{LB}$ edited and revised the 
manuscript. All authors have substantially contributed to the article and approved the submitted version.

\section{FUNDING}

This work was supported by the National Natural Science Foundation of China (grant numbers 81772420, 81272050, and 31900847); the China Postdoctoral Fund (grant number

\section{REFERENCES}

Abed, É, Delalandre, A., and Lajeunesse, D. (2017). Beneficial effect of resveratrol on phenotypic features and activity of osteoarthritic osteoblasts. Arthritis Res. Ther. 19:151.

Adkar, S. S., Brunger, J. M., Willard, V. P., Wu, C. L., Gersbach, C. A., and Guilak, F. (2017). Genome engineering for personalized arthritis therapeutics. Trends Mol. Med. 23, 917-931. doi: 10.1016/j.molmed.2017.08.002

Alsalameh, S., Amin, R., Gemba, T., and Lotz, M. (2004). Identification of mesenchymal progenitor cells in normal and osteoarthritic human articular cartilage. Arthritis Rheum. 50, 1522-1532. doi: 10.1002/art.20269

Anderson, H. C. (2003). Matrix vesicles and calcification. Curr. Rheumatol. Rep. 5, 222-226.

Asghar, S., Litherland, G. J., Lockhart, J. C., Goodyear, C. S., and Crilly, A. (2020). Exosomes in intercellular communication and implications for osteoarthritis. Rheumatology (Oxford) 59, 57-68.

Assoian, R. K., Fleurdelys, B. E., Stevenson, H. C., Miller, P. J., Madtes, D. K., Raines, E. W., et al. (1987). Expression and secretion of type beta transforming growth factor by activated human macrophages. Proc. Natl. Acad. Sci. U.S.A. 84, 6020-6024. doi: 10.1073/pnas.84.17.6020

Attur, M., Samuels, J., Krasnokutsky, S., and Abramson, S. B. (2010). Targeting the synovial tissue for treating osteoarthritis $(\mathrm{OA})$ : where is the evidence? Best Pract. Res. Clin. Rheumatol. 24, 71-79. doi: 10.1016/j.berh.2009.08.011

Aulin, C., Lassacher, T., Palmblad, K., and Erlandsson Harris, H. (2020). Early stage blockade of the alarmin HMGB1 reduces cartilage destruction in experimental OA. Osteoarthritis Cartilage 28, 698-707. doi: 10.1016/j.joca.2020. 01.003

Bannuru, R. R., Osani, M. C., Vaysbrot, E. E., Arden, N. K., Bennell, K., BiermaZeinstra, S. M. A., et al. (2019). OARSI guidelines for the non-surgical management of knee, hip, and polyarticular osteoarthritis. Osteoarthritis Cartilage 27, 1578-1589. doi: 10.1016/j.joca.2019.06.011

Barton, K. I., Shekarforoush, M., Heard, B. J., Sevick, J. L., Vakil, P., Atarod, M., et al. (2017). Use of pre-clinical surgically induced models to understand biomechanical and biological consequences of PTOA development. J. Orthop. Res. 35, 454-465. doi: 10.1002/jor.23322

Bastiaansen-Jenniskens, Y. M., Wei, W., Feijt, C., Waarsing, J. H., Verhaar, J. A., Zuurmond, A. M., et al. (2013). Stimulation of fibrotic processes by the infrapatellar fat pad in cultured synoviocytes from patients with osteoarthritis: a possible role for prostaglandin f2 $\alpha$. Arthritis Rheum. 65, 2070-2080. doi: $10.1002 /$ art.37996

Beekhuizen, M., Bastiaansen-Jenniskens, Y. M., Koevoet, W., Saris, D. B., Dhert, W. J., Creemers, L. B., et al. (2011). Osteoarthritic synovial tissue inhibition of proteoglycan production in human osteoarthritic knee cartilage: establishment and characterization of a long-term cartilage-synovium coculture. Arthritis Rheum. 63, 1918-1927. doi: 10.1002/art.30364

Behrends, D. A., Cheng, L., Sullivan, M. B., Wang, M. H., Roby, G. B., Zayed, N., et al. (2014). Defective bone repair in mast cell deficient mice with c-Kit loss of function. Eur. Cells Mater. 28, 209-221; discussion 21-22.

Bhosale, A. M., and Richardson, J. B. (2008). Articular cartilage: structure, injuries and review of management. Br. Med. Bull. 87, 77-95. doi: 10.1093/bmb/ldn025

Biosse-Duplan, M., Baroukh, B., Dy, M., de Vernejoul, M. C., and Saffar, J. L. (2009). Histamine promotes osteoclastogenesis through the differential expression of histamine receptors on osteoclasts and osteoblasts. Am. J. Pathol. 174, 1426-1434. doi: 10.2353/ajpath.2009.080871

Bjørge, I. M., Kim, S. Y., Mano, J. F., Kalionis, B., and Chrzanowski, W. (2017). Extracellular vesicles, exosomes and shedding vesicles in regenerative medicine
2019M66169); and the Liaoning Provincial Doctor Start-up Fund (grant number 2019JH3/10100299).

\section{ACKNOWLEDGMENTS}

We thank the laboratory affiliated to the China Medical University for the core support.

- a new paradigm for tissue repair. Biomater. Sci. 6, 60-78. doi: 10.1039/ c7bm00479f

Blanco, F. J., Valdes, A. M., and Rego-Pérez, I. (2018). Mitochondrial DNA variation and the pathogenesis of osteoarthritis phenotypes. Nat. Rev. Rheumatol. 14, 327-340. doi: 10.1038/s41584-018-0001-0

Bondeson, J., Blom, A. B., Wainwright, S., Hughes, C., Caterson, B., and van den Berg, W. B. (2010). The role of synovial macrophages and macrophageproduced mediators in driving inflammatory and destructive responses in osteoarthritis. Arthritis Rheum. 62, 647-657. doi: 10.1002/art.27290

Bosserhoff, A. K., Hofmeister, S., Ruedel, A., and Schubert, T. (2014). DCC is expressed in a CD166-positive subpopulation of chondrocytes in human osteoarthritic cartilage and modulates CRE activity. Int. J. Clin. Exp. Pathol. 7, 1947-1956.

Brandt, K. D., Radin, E. L., Dieppe, P. A., and van de Putte, L. (2006). Yet more evidence that osteoarthritis is not a cartilage disease. Ann. Rheum. Dis. 65, 1261-1264. doi: 10.1136/ard.2006.058347

Bridges, A. J., Malone, D. G., Jicinsky, J., Chen, M., Ory, P., Engber, W., et al. (1991). Human synovial mast cell involvement in rheumatoid arthritis and osteoarthritis. Relationship to disease type, clinical activity, and antirheumatic therapy. Arthritis Rheum. 34, 1116-1124. doi: 10.1002/art.1780340907

Brittberg, M., Lindahl, A., Nilsson, A., Ohlsson, C., Isaksson, O., and Peterson, L. (1994). Treatment of deep cartilage defects in the knee with autologous chondrocyte transplantation. N. Engl. J. Med. 331, 889-895.

Brunger, J. M., Zutshi, A., Willard, V. P., Gersbach, C. A., and Guilak, F. (2017). Genome engineering of stem cells for autonomously regulated, closed-loop delivery of biologic drugs. Stem Cell Rep. 8, 1202-1213. doi: 10.1016/j.stemcr. 2017.03.022

Buckley, C. T., Vinardell, T., and Kelly, D. J. (2010). Oxygen tension differentially regulates the functional properties of cartilaginous tissues engineered from infrapatellar fat pad derived MSCs and articular chondrocytes. Osteoarthritis Cartilage 18, 1345-1354. doi: 10.1016/j.joca.2010.07.004

Buckley, M. G., Walters, C., Wong, W. M., Cawley, M. I., Ren, S., Schwartz, L. B., et al. (1997). Mast cell activation in arthritis: detection of alpha- and betatryptase, histamine and eosinophil cationic protein in synovial fluid. Clin. Sci. (Lond. Engl. 1979) 93, 363-370. doi: 10.1042/cs0930363

Buhrmann, C., Mobasheri, A., Matis, U., and Shakibaei, M. (2010). Curcumin mediated suppression of nuclear factor- $\kappa \mathrm{B}$ promotes chondrogenic differentiation of mesenchymal stem cells in a high-density co-culture microenvironment. Arthritis Res. Ther. 12:R127.

Burr, D. B. (1998). The importance of subchondral bone in osteoarthrosis. Curr. Opin. Rheumatol. 10, 256-262. doi: 10.1097/00002281-199805000-00017

Cao, Y., Jansen, I. D., Sprangers, S., Stap, J., Leenen, P. J., Everts, V., et al. (2016). IL-1 $\beta$ differently stimulates proliferation and multinucleation of distinct mouse bone marrow osteoclast precursor subsets. J. Leukoc. Biol. 100, 513-523. doi: 10.1189/jlb.1a1215-543r

Castañeda, S., Roman-Blas, J. A., Largo, R., and Herrero-Beaumont, G. (2012). Subchondral bone as a key target for osteoarthritis treatment. Biochem. Pharmacol. 83, 315-323. doi: 10.1016/j.bcp.2011.09.018

Champagne, C. M., Takebe, J., Offenbacher, S., and Cooper, L. F. (2002). Macrophage cell lines produce osteoinductive signals that include bone morphogenetic protein-2. Bone 30, 26-31. doi: 10.1016/s8756-3282(01)00 638-x

Chen, S., Fu, P., Wu, H., and Pei, M. (2017). Meniscus, articular cartilage and nucleus pulposus: a comparative review of cartilage-like tissues in anatomy, development and function. Cell Tissue Res. 370, 53-70. doi: 10.1007/s00441017-2613-0 
Chen, Y., Wang, T., Guan, M., Zhao, W., Leung, F. K., Pan, H., et al. (2015). Bone turnover and articular cartilage differences localized to subchondral cysts in knees with advanced osteoarthritis. Osteoarthritis Cartilage 23, 2174-2183. doi: 10.1016/j.joca.2015.07.012

Chen, Y., Xue, K., Zhang, X., Zheng, Z., and Liu, K. (2018). Exosomes derived from mature chondrocytes facilitate subcutaneous stable ectopic chondrogenesis of cartilage progenitor cells. Stem Cell Res. Ther. 9:318.

Chen, Z., Ma, Y., Li, X., Deng, Z., Zheng, M., and Zheng, Q. (2020). the immune cell landscape in different anatomical structures of knee in osteoarthritis: a gene expression-based study. Biomed. Res. Int. 2020:9647072.

Clarke, B. (2008). Normal bone anatomy and physiology. Clin. J. Am. Soc. Nephrol. CJASN 3 (Suppl. 3), S131-S139.

Clockaerts, S., Bastiaansen-Jenniskens, Y. M., Runhaar, J., Van Osch, G. J., Van Offel, J. F., Verhaar, J. A., et al. (2010). The infrapatellar fat pad should be considered as an active osteoarthritic joint tissue: a narrative review. Osteoarthritis Cartilage 18, 876-882. doi: 10.1016/j.joca.2010. 03.014

Cooper, A. M., and Khader, S. A. (2007). IL-12p40: an inherently agonistic cytokine. Trends Immunol. 28, 33-38. doi: 10.1016/j.it.2006.11.002

Corrado, A., Neve, A., and Cantatore, F. P. (2013). Expression of vascular endothelial growth factor in normal, osteoarthritic and osteoporotic osteoblasts. Clin. Exp. Med. 13, 81-84. doi: 10.1007/s10238-011-0170-5

Correa, D., Somoza, R. A., Lin, P., Greenberg, S., Rom, E., Duesler, L., et al. (2015). Sequential exposure to fibroblast growth factors (FGF) 2, 9 and 18 enhances hMSC chondrogenic differentiation. Osteoarthritis Cartilage 23, 443-453. doi: 10.1016/j.joca.2014.11.013

Cosenza, S., Ruiz, M., Toupet, K., Jorgensen, C., and Noël, D. (2017). Mesenchymal stem cells derived exosomes and microparticles protect cartilage and bone from degradation in osteoarthritis. Sci. Rep. 7:16214.

Cosenza, S., Toupet, K., Maumus, M., Luz-Crawford, P., Blanc-Brude, O., Jorgensen, C., et al. (2018). Mesenchymal stem cells-derived exosomes are more immunosuppressive than microparticles in inflammatory arthritis. Theranostics 8, 1399-1410. doi: 10.7150/thno.21072

Culemann, S., Grüneboom, A., Nicolás-Ávila, J., Weidner, D., Lämmle, K. F., Rothe, T., et al. (2019). Locally renewing resident synovial macrophages provide a protective barrier for the joint. Nature 572, 670-675. doi: 10.1038/s41586019-1471-1

Cutolo, M., Berenbaum, F., Hochberg, M., Punzi, L., and Reginster, J. Y. (2015). Commentary on recent therapeutic guidelines for osteoarthritis. Semin. Arthritis Rheum. 44, 611-617. doi: 10.1016/j.semarthrit.2014.12.003

Damia, E., Chicharro, D., Lopez, S., Cuervo, B., Rubio, M., Sopena, J. J., et al. (2018). Adipose-Derived mesenchymal stem cells: are they a good therapeutic strategy for osteoarthritis? Int. J. Mol. Sci. 19:1926. doi: 10.3390/ijms19071926

De Bari, C., and Roelofs, A. J. (2018). Stem cell-based therapeutic strategies for cartilage defects and osteoarthritis. Curr. Opin. Pharmacol. 40, 74-80. doi: 10.1016/j.coph.2018.03.009

De Jong, O. G., Van Balkom, B. W., Schiffelers, R. M., Bouten, C. V., and Verhaar, M. C. (2014). Extracellular vesicles: potential roles in regenerative medicine. Front. Immunol. 5:608. doi: 10.3389/fimmu.2014.00608

de Jong, O. G., Verhaar, M. C., Chen, Y., Vader, P., Gremmels, H., Posthuma, G., et al. (2012). Cellular stress conditions are reflected in the protein and RNA content of endothelial cell-derived exosomes. J. Extracell. Vesicles 1:18396. doi: 10.3402/jev.v1i0.18396

de Lange-Brokaar, B. J., Ioan-Facsinay, A., van Osch, G. J., Zuurmond, A. M., Schoones, J., Toes, R. E., et al. (2012). Synovial inflammation, immune cells and their cytokines in osteoarthritis: a review. Osteoarthritis Cartilage 20, 1484-1499. doi: 10.1016/j.joca.2012.08.027

Di Matteo, B., Vandenbulcke, F., Vitale, N. D., Iacono, F., Ashmore, K., Marcacci, M., et al. (2019). Minimally manipulated mesenchymal stem cells for the treatment of knee osteoarthritis: a systematic review of clinical evidence. Stem Cells Int. 2019:1735242.

Dickson, B. M., Roelofs, A. J., Rochford, J. J., Wilson, H. M., and De Bari, C. (2019). The burden of metabolic syndrome on osteoarthritic joints. Arthritis Res. Ther. 21:289.

Diekman, B. O., Christoforou, N., Willard, V. P., Sun, H., Sanchez-Adams, J., Leong, K. W., et al. (2012). Cartilage tissue engineering using differentiated and purified induced pluripotent stem cells. Proc. Natl. Acad. Sci. U.S.A. 109, 19172-19177. doi: 10.1073/pnas. 1210422109
Dieppe, P., Lim, K., and Lohmander, S. (2011). Who should have knee joint replacement surgery for osteoarthritis? Int. J. Rheum. Dis. 14, 175-180. doi: 10.1111/j.1756-185x.2011.01611.x

Ding, D. C., Wu, K. C., Chou, H. L., Hung, W. T., Liu, H. W., and Chu, T. Y. (2015). Human infrapatellar fat pad-derived stromal cells have more potent differentiation capacity than other mesenchymal cells and can be enhanced by hyaluronan. Cell Transplant. 24, 1221-1232. doi: 10.3727/096368914x681937

Dragoo, J. L., Johnson, C., and McConnell, J. (2012). Evaluation and treatment of disorders of the infrapatellar fat pad. Sports Med. (Auckland, NZ) 42, 51-67. doi: 10.2165/11595680-000000000-00000

Dutta, B., Arya, R. K., Goswami, R., Alharbi, M. O., Sharma, S., and Rahaman, S. O. (2020). Role of macrophage TRPV4 in inflammation. Lab. Invest. J. Tech. Methods Pathol. 100, 178-185. doi: 10.1038/s41374-019-0334-6

Enomoto, T., Akagi, R., Ogawa, Y., Yamaguchi, S., Hoshi, H., Sasaki, T., et al. (2020). Timing of intra-articular injection of synovial mesenchymal stem cells affects cartilage restoration in a partial thickness cartilage defect model in rats. Cartilage 11, 122-129. doi: 10.1177/1947603518786542

Eymard, F., Pigenet, A., Citadelle, D., Flouzat-Lachaniette, C. H., Poignard, A., Benelli, C., et al. (2014). Induction of an inflammatory and prodegradative phenotype in autologous fibroblast-like synoviocytes by the infrapatellar fat pad from patients with knee osteoarthritis. Arthritis Rheumatol. 66, 2165-2174. doi: 10.1002/art.38657

Falconer, J., Murphy, A. N., Young, S. P., Clark, A. R., Tiziani, S., Guma, M., et al. (2018). Review: synovial cell metabolism and chronic inflammation in rheumatoid arthritis. Arthritis Rheumatol. 70, 984-999. doi: 10.1002/art.40504

Favero, M., El-Hadi, H., Belluzzi, E., Granzotto, M., Porzionato, A., Sarasin, G., et al. (2017). Infrapatellar fat pad features in osteoarthritis: a histopathological and molecular study. Rheumatology (Oxford) 56, 1784-1793. doi: 10.1093/ rheumatology/kex287

Feng, X., Li, Z., Wei, J., Feng, Z., Wu, W., and Zhao, Y. (2018). Injectable cartilaginous template transformed BMSCs into vascularized bone. Sci. Rep. 8:8244.

Fernandes, T. L., Gomoll, A. H., Lattermann, C., Hernandez, A. J., Bueno, D. F., and Amano, M. T. (2020). Macrophage: a potential target on cartilage regeneration. Front. Immunol. 11:111. doi: 10.3389/fimmu.2020.00111

Fickert, S., Fiedler, J., and Brenner, R. E. (2004). Identification of subpopulations with characteristics of mesenchymal progenitor cells from human osteoarthritic cartilage using triple staining for cell surface markers. Arthritis Res. Ther. 6, R422-R432.

Firestein, G. S., and McInnes, I. B. (2017). Immunopathogenesis of rheumatoid arthritis. Immunity 46, 183-196. doi: 10.1016/j.immuni.2017.02.006

Fujisawa, T., Hattori, T., Takahashi, K., Kuboki, T., Yamashita, A., and Takigawa, M. (1999). Cyclic mechanical stress induces extracellular matrix degradation in cultured chondrocytes via gene expression of matrix metalloproteinases and interleukin-1. J. Biochem. 125, 966-975. doi: 10.1093/oxfordjournals.jbchem. a022376

Galipeau, J., Krampera, M., Barrett, J., Dazzi, F., Deans, R. J., DeBruijn, J., et al. (2016). International Society for Cellular Therapy perspective on immune functional assays for mesenchymal stromal cells as potency release criterion for advanced phase clinical trials. Cytotherapy 18, 151-159. doi: 10.1016/j.jcyt. 2015.11.008

Galli, S. J., and Tsai, M. (2012). IgE and mast cells in allergic disease. Nat. Med. 18, 693-704. doi: 10.1038/nm.2755

Gao, M., Gao, W., Papadimitriou, J. M., Zhang, C., Gao, J., and Zheng, M. (2018). Exosomes-the enigmatic regulators of bone homeostasis. Bone Res. 6:36.

GBD 2017 Risk Factor Collaborators (2018). Global, regional, and national comparative risk assessment of 84 behavioural, environmental and occupational, and metabolic risks or clusters of risks for 195 countries and territories, 1990-2017: a systematic analysis for the Global Burden of Disease Study 2017. Lancet 392, 1923-1994.

Genin, M., Clement, F., Fattaccioli, A., Raes, M., and Michiels, C. (2015). M1 and M2 macrophages derived from THP-1 cells differentially modulate the response of cancer cells to etoposide. BMC Cancer 15:577. doi: 10.1186/s12885-0151546-9

Gerace, D., Martiniello-Wilks, R., Nassif, N. T., Lal, S., Steptoe, R., and Simpson, A. M. (2017). CRISPR-targeted genome editing of mesenchymal stem cellderived therapies for type 1 diabetes: a path to clinical success? Stem Cell Res. Ther. 8:62. 
Gibson, J. D., O'Sullivan, M. B., Alaee, F., Paglia, D. N., Yoshida, R., Guzzo, R. M., et al. (2017). Regeneration of articular cartilage by human ESC-Derived mesenchymal progenitors treated sequentially with BMP-2 and Wnt5a. Stem Cells Transl. Med. 6, 40-50. doi: 10.5966/sctm.2016-0020

Glass, K. A., Link, J. M., Brunger, J. M., Moutos, F. T., Gersbach, C. A., and Guilak, F. (2014). Tissue-engineered cartilage with inducible and tunable immunomodulatory properties. Biomaterials 35, 5921-5931. doi: 10.1016/j. biomaterials.2014.03.073

Gore, M., Tai, K. S., Sadosky, A., Leslie, D., and Stacey, B. R. (2011). Clinical comorbidities, treatment patterns, and direct medical costs of patients with osteoarthritis in usual care: a retrospective claims database analysis. J. Med. Econ. 14, 497-507. doi: 10.3111/13696998.2011.59 4347

Guo, S. C., Tao, S. C., Yin, W. J., Qi, X., Sheng, J. G., and Zhang, C. Q. (2016). Exosomes from human synovial-derived mesenchymal stem cells prevent glucocorticoid-induced osteonecrosis of the femoral head in the rat. Int. J. Biol. Sci. 12, 1262-1272. doi: 10.7150/ijbs. 16150

Hamilton, A. M., Cheung, W. Y., Gómez-Aristizábal, A., Sharma, A., Nakamura, S., Chaboureau, A., et al. (2019). Iron nanoparticle-labeled murine mesenchymal stromal cells in an osteoarthritic model persists and suggests anti-inflammatory mechanism of action. PLoS One 14:e0214107. doi: 10.1371/journal.pone. 0214107

Harrell, C. R., Markovic, B. S., Fellabaum, C., Arsenijevic, A., and Volarevic, V. (2019). Mesenchymal stem cell-based therapy of osteoarthritis: current knowledge and future perspectives. Biomed. Pharmacother. 109, 2318-2326. doi: 10.1016/j.biopha.2018.11.099

Headland, S. E., Jones, H. R., Norling, L. V., Kim, A., Souza, P. R., Corsiero, E., et al. (2015). Neutrophil-derived microvesicles enter cartilage and protect the joint in inflammatory arthritis. Sci. Transl. Med. 7:315ra190. doi: 10.1126/scitranslmed. aac5608

Heldring, N., Mäger, I., Wood, M. J., Le Blanc, K., and Andaloussi, S. E. (2015). Therapeutic potential of multipotent mesenchymal stromal cells and their extracellular vesicles. Human Gene Ther. 26, 506-517. doi: 10.1089/hum.2015. 072

Hendriks, J., Riesle, J., and van Blitterswijk, C. A. (2007). Co-culture in cartilage tissue engineering. J. Tissue Eng. Regen. Med. 1, 170-178. doi: 10.1002/term.19

Henrotin, Y., Pesesse, L., and Sanchez, C. (2012). Subchondral bone and osteoarthritis: biological and cellular aspects. Osteoporos. Int. 23(Suppl. 8), S847-S851.

Hilal, G., Martel-Pelletier, J., Pelletier, J. P., Ranger, P., and Lajeunesse, D. (1998). Osteoblast-like cells from human subchondral osteoarthritic bone demonstrate an altered phenotype in vitro: possible role in subchondral bone sclerosis. Arthritis Rheum. 41, 891-899. doi: 10.1002/1529-0131(199805)41:5<891::aid$\operatorname{art17}>3.0 . c 0 ; 2-\mathrm{x}$

Hoshi, H., Akagi, R., Yamaguchi, S., Muramatsu, Y., Akatsu, Y., Yamamoto, Y., et al. (2017). Effect of inhibiting MMP13 and ADAMTS5 by intra-articular injection of small interfering RNA in a surgically induced osteoarthritis model of mice. Cell Tissue Res. 368, 379-387. doi: 10.1007/s00441-016-2563-y

Hunter, D. J., and Bierma-Zeinstra, S. (2019). Osteoarthritis. Lancet 393, 17451759.

Ioan-Facsinay, A., Kwekkeboom, J. C., Westhoff, S., Giera, M., Rombouts, Y., van Harmelen, V., et al. (2013). Adipocyte-derived lipids modulate CD4+ T-cell function. Eur. J. Immunol. 43, 1578-1587. doi: 10.1002/eji.201243096

Jaiswal, R., Raymond Grau, G. E., and Bebawy, M. (2014). Cellular communication via microparticles: role in transfer of multidrug resistance in cancer. Future Oncol. (Lond. Engl.) 10, 655-669. doi: 10.2217/fon.13.230

Ji, Q., Zheng, Y., Zhang, G., Hu, Y., Fan, X., Hou, Y., et al. (2019). Single-cell RNAseq analysis reveals the progression of human osteoarthritis. Ann. Rheum. Dis. 78, 100-110. doi: 10.1136/annrheumdis-2017-212863

Jin, Z., Ren, J., and Qi, S. (2020). Human bone mesenchymal stem cells-derived exosomes overexpressing microRNA-26a-5p alleviate osteoarthritis via downregulation of PTGS2. Int. Immunopharmacol. 78:105946. doi: 10.1016/j.intimp. 2019.105946

Jo, C. H., Chai, J. W., Jeong, E. C., Oh, S., Shin, J. S., Shim, H., et al. (2017). Intraarticular injection of mesenchymal stem cells for the treatment of osteoarthritis of the knee: a 2-Year follow-up study. Am. J. Sports Med. 45, 2774-2783. doi: $10.1177 / 0363546517716641$
Jubeck, B., Gohr, C., Fahey, M., Muth, E., Matthews, M., Mattson, E., et al. (2008). Promotion of articular cartilage matrix vesicle mineralization by type I collagen. Arthritis Rheum. 58, 2809-2817. doi: 10.1002/art.23762

Jung, Y. K., Han, M. S., Park, H. R., Lee, E. J., Jang, J. A., Kim, G. W., et al. (2018). Calcium-phosphate complex increased during subchondral bone remodeling affects earlystage osteoarthritis. Sci. Rep. 8:487.

Kang, Y., Song, J., Kim, D., Ahn, C., Park, S., Chun, C. H., et al. (2016). PCGEM1 stimulates proliferation of osteoarthritic synoviocytes by acting as a sponge for miR-770. J. Orthop. Res. 34, 412-418. doi: 10.1002/jor.23046

Kato, T., Miyaki, S., Ishitobi, H., Nakamura, Y., Nakasa, T., Lotz, M. K., et al. (2014). Exosomes from IL-1 $\beta$ stimulated synovial fibroblasts induce osteoarthritic changes in articular chondrocytes. Arthritis Res. Ther. 16:R163.

Katsimbri, P. (2017). The biology of normal bone remodelling. Eur. J. Cancer Care 26:e12740. doi: 10.1111/ecc.12740

Kim, S. H., Djaja, Y. P., Park, Y. B., Park, J. G., Ko, Y. B., and Ha, C. W. (2020). Intra-articular injection of culture-expanded mesenchymal stem cells without adjuvant surgery in knee osteoarthritis: a systematic review and meta-analysis. Am. J. Sports Med. 48, 2839-2849. doi: 10.1177/0363546519892278

Kim, Y. S., and Koh, Y. G. (2018). Comparative matched-pair analysis of openwedge high tibial osteotomy with versus without an injection of adipose-derived mesenchymal stem cells for varus knee osteoarthritis: clinical and secondlook arthroscopic results. Am. J. Sports Med. 46, 2669-2677. doi: 10.1177/ 0363546518785973

Klein-Wieringa, I. R., Andersen, S. N., Kwekkeboom, J. C., Giera, M., de LangeBrokaar, B. J., van Osch, G. J., et al. (2013). Adipocytes modulate the phenotype of human macrophages through secreted lipids. J. Immunol. 191, 1356-1363. doi: 10.4049/jimmunol.1203074

Kloppenburg, M., and Berenbaum, F. (2020). Osteoarthritis year in review 2019: epidemiology and therapy. Osteoarthritis Cartilage 28, 242-248. doi: 10.1016/j. joca.2020.01.002

Kobayashi, K., Takahashi, N., Jimi, E., Udagawa, N., Takami, M., Kotake, S., et al. (2000). Tumor necrosis factor alpha stimulates osteoclast differentiation by a mechanism independent of the ODF/RANKL-RANK interaction. J. Exp. Med. 191, 275-286. doi: 10.1084/jem.191.2.275

Koelling, S., Kruegel, J., Irmer, M., Path, J. R., Sadowski, B., Miro, X., et al. (2009). Migratory chondrogenic progenitor cells from repair tissue during the later stages of human osteoarthritis. Cell Stem Cell 4, 324-335. doi: 10.1016/j.stem. 2009.01.015

Koh, Y. G., and Choi, Y. J. (2012). Infrapatellar fat pad-derived mesenchymal stem cell therapy for knee osteoarthritis. Knee 19, 902-907. doi: 10.1016/j.knee.2012. 04.001

Koizumi, K., Ebina, K., Hart, D. A., Hirao, M., Noguchi, T., Sugita, N., et al. (2016). Synovial mesenchymal stem cells from osteo- or rheumatoid arthritis joints exhibit good potential for cartilage repair using a scaffold-free tissue engineering approach. Osteoarthritis Cartilage 24, 1413-1422. doi: 10.1016/j. joca.2016.03.006

Kondo, S., Nakagawa, Y., Mizuno, M., Katagiri, K., Tsuji, K., Kiuchi, S., et al. (2019). Transplantation of aggregates of autologous synovial mesenchymal stem cells for treatment of cartilage defects in the femoral condyle and the femoral groove in microminipigs. Am. J. Sports Med. 47, 2338-2347. doi: $10.1177 / 0363546519859855$

Kouroupis, D., Bowles, A. C., Willman, M. A., Perucca Orfei, C., Colombini, A., Best, T. M., et al. (2019). Infrapatellar fat pad-derived MSC response to inflammation and fibrosis induces an immunomodulatory phenotype involving CD10-mediated Substance P degradation. Sci. Rep. 9:10864.

Kreutz, M., Andreesen, R., Krause, S. W., Szabo, A., Ritz, E., and Reichel, H. (1993). 1,25-dihydroxyvitamin D3 production and vitamin D3 receptor expression are developmentally regulated during differentiation of human monocytes into macrophages. Blood 82, 1300-1307. doi: 10.1182/blood.v82.4.1300.1300

Kroner, J., Kovtun, A., Kemmler, J., Messmann, J. J., Strauss, G., Seitz, S., et al. (2017). Mast cells are critical regulators of bone fracture-induced inflammation and osteoclast formation and activity. J. Bone Min. Res. 32, 2431-2444. doi: $10.1002 / \mathrm{jbmr} .3234$

Kuang, L., Wu, J., Su, N., Qi, H., Chen, H., Zhou, S., et al. (2020). FGFR3 deficiency enhances CXCL12-dependent chemotaxis of macrophages via upregulating CXCR7 and aggravates joint destruction in mice. Ann. Rheum. Dis. 79, 112-122. doi: 10.1136/annrheumdis-2019-215696 
Kurth, T., Hedbom, E., Shintani, N., Sugimoto, M., Chen, F. H., Haspl, M., et al. (2007). Chondrogenic potential of human synovial mesenchymal stem cells in alginate. Osteoarthritis Cartilage 15, 1178-1189. doi: 10.1016/j.joca.2007.03.015

Kwan Tat, S., Pelletier, J. P., Lajeunesse, D., Fahmi, H., Lavigne, M., and MartelPelletier, J. (2008). The differential expression of osteoprotegerin (OPG) and receptor activator of nuclear factor kappaB ligand (RANKL) in human osteoarthritic subchondral bone osteoblasts is an indicator of the metabolic state of these disease cells. Clin. Exp. Rheumatol. 26, 295-304.

Lach, M., Trzeciak, T., Richter, M., Pawlicz, J., and Suchorska, W. M. (2014). Directed differentiation of induced pluripotent stem cells into chondrogenic lineages for articular cartilage treatment. J. Tissue Eng. 5:2041731414552701.

Lamichhane, T. N., Sokic, S., Schardt, J. S., Raiker, R. S., Lin, J. W., and Jay, S. M. (2015). Emerging roles for extracellular vesicles in tissue engineering and regenerative medicine. Tissue Eng. Part B Rev. 21, 45-54. doi: 10.1089/ten.teb. 2014.0300

Lee, H., Kashiwakura, J., Matsuda, A., Watanabe, Y., Sakamoto-Sasaki, T., Matsumoto, K., et al. (2013). Activation of human synovial mast cells from rheumatoid arthritis or osteoarthritis patients in response to aggregated IgG through Fc $\gamma$ receptor I and Fc $\gamma$ receptor II. Arthritis Rheum. 65, 109-119. doi: 10.1002/art.37741

Lee, M. J., Kim, J., Kim, M. Y., Bae, Y. S., Ryu, S. H., Lee, T. G., et al. (2010). Proteomic analysis of tumor necrosis factor-alpha-induced secretome of human adipose tissue-derived mesenchymal stem cells. J. Proteome Res. 9, 1754-1762. doi: $10.1021 /$ pr900898n

Lee, W. S., Kim, H. J., Kim, K. I., Kim, G. B., and Jin, W. (2019). Intra-Articular injection of autologous adipose tissue-derived mesenchymal stem cells for the treatment of knee osteoarthritis: a phase IIB, randomized, placebo-controlled clinical trial. Stem Cells Transl. Med. 8, 504-511. doi: 10.1002/sctm.18-0122

Lee, W. Y., and Wang, B. (2017). Cartilage repair by mesenchymal stem cells: clinical trial update and perspectives. J. Orthop. Transl. 9, 76-88. doi: 10.1016/ j.jot.2017.03.005

Levy, O., Kuai, R., Siren, E. M. J., Bhere, D., Milton, Y., Nissar, N., et al. (2020). Shattering barriers toward clinically meaningful MSC therapies. Sci. Adv. 6:eaba6884. doi: 10.1126/sciadv.aba6884

Li, C. J., Xiao, Y., Yang, M., Su, T., Sun, X., Guo, Q., et al. (2018). Long noncoding RNA Bmncr regulates mesenchymal stem cell fate during skeletal aging. J. Clin. Invest. 128, 5251-5266. doi: 10.1172/jci99044

Li, D., Liu, J., Guo, B., Liang, C., Dang, L., Lu, C., et al. (2016). Osteoclast-derived exosomal miR-214-3p inhibits osteoblastic bone formation. Nat. Commun. 7:10872.

Li, G., Yin, J., Gao, J., Cheng, T. S., Pavlos, N. J., Zhang, C., et al. (2013). Subchondral bone in osteoarthritis: insight into risk factors and microstructural changes. Arthritis Res. Ther. 15:223. doi: 10.1186/ar4405

Li, J., Ding, Z., Li, Y., Wang, W., Wang, J., Yu, H., et al. (2020). BMSCsDerived exosomes ameliorate pain via abrogation of aberrant nerve invasion in subchondral bone in lumbar facet joint osteoarthritis. J. Orthop. Res. 38, 670-679. doi: 10.1002/jor.24497

Li, X., Guo, W., Zha, K., Jing, X., Wang, M., Zhang, Y., et al. (2019). Enrichment of CD146(+) adipose-derived stem cells in combination with articular cartilage extracellular matrix scaffold promotes cartilage regeneration. Theranostics 9, 5105-5121. doi: 10.7150/thno.33904

Li, Z., Wang, Y., Xiao, K., Xiang, S., Li, Z., and Weng, X. (2018). Emerging role of exosomes in the joint diseases. Cell. Physiol. Biochem. 47, 2008-2017. doi: $10.1159 / 000491469$

Lietman, S. A. (2016). Induced pluripotent stem cells in cartilage repair. World J. Orthop. 7, 149-155. doi: 10.5312/wjo.v7.i3.149

Lin, C., Liu, L., Zeng, C., Cui, Z. K., Chen, Y., Lai, P., et al. (2019). Activation of mTORC1 in subchondral bone preosteoblasts promotes osteoarthritis by stimulating bone sclerosis and secretion of CXCL12. Bone Res. 7:5.

Liu, B., Zhang, M., Zhao, J., Zheng, M., and Yang, H. (2018). Imbalance of M1/M2 macrophages is linked to severity level of knee osteoarthritis. Exp. Ther. Med. 16, 5009-5014.

Liu, C., and Su, C. (2019). Design strategies and application progress of therapeutic exosomes. Theranostics 9, 1015-1028. doi: 10.7150/thno.30853

Liu, C., Li, Y., Yang, Z., Zhou, Z., Lou, Z., and Zhang, Q. (2020). Kartogenin enhances the therapeutic effect of bone marrow mesenchymal stem cells derived exosomes in cartilage repair. Nanomedicine (Lond. Engl.) 15, 273-288. doi: 10.2217/nnm-2019-0208
Liu, X., Yang, Y., Li, Y., Niu, X., Zhao, B., Wang, Y., et al. (2017). Integration of stem cell-derived exosomes with in situ hydrogel glue as a promising tissue patch for articular cartilage regeneration. Nanoscale 9, 4430-4438. doi: 10.1039/ c7nr00352h

Lo Sicco, C., Reverberi, D., Balbi, C., Ulivi, V., Principi, E., Pascucci, L., et al. (2017). Mesenchymal stem cell-derived extracellular vesicles as mediators of anti-inflammatory effects: endorsement of macrophage polarization. Stem Cells Transl. Med. 6, 1018-1028. doi: 10.1002/sctm.16-0363

Loeser, R. F. (2009). Aging and osteoarthritis: the role of chondrocyte senescence and aging changes in the cartilage matrix. Osteoarthritis Cartilage 17, 971-979. doi: 10.1016/j.joca.2009.03.002

Loeser, R. F., Olex, A. L., McNulty, M. A., Carlson, C. S., Callahan, M. F., Ferguson, C. M., et al. (2012). Microarray analysis reveals age-related differences in gene expression during the development of osteoarthritis in mice. Arthritis Rheum. 64, 705-717. doi: 10.1002/art.33388

Löfvall, H., Newbould, H., Karsdal, M. A., Dziegiel, M. H., Richter, J., Henriksen, K., et al. (2018). Osteoclasts degrade bone and cartilage knee joint compartments through different resorption processes. Arthritis Res. Ther. 20:67.

Ma, J., Zhao, Y., Sun, L., Sun, X., Zhao, X., Sun, X., et al. (2017). Exosomes derived from Akt-modified human umbilical cord mesenchymal stem cells improve cardiac regeneration and promote angiogenesis via activating platelet-derived growth factor D. Stem Cells Transl. Med. 6, 51-59. doi: 10.5966/sctm.2016-0038

Malda, J., Boere, J., van de Lest, C. H., van Weeren, P., and Wauben, M. H. (2016). Extracellular vesicles - new tool for joint repair and regeneration. Nat. Rev. Rheumatol. 12, 243-249. doi: 10.1038/nrrheum.2015.170

Mamidi, M. K., Das, A. K., Zakaria, Z., and Bhonde, R. (2016). Mesenchymal stromal cells for cartilage repair in osteoarthritis. Osteoarthritis Cartilage 24, 1307-1316. doi: 10.1016/j.joca.2016.03.003

Mao, G., Zhang, Z., Hu, S., Zhang, Z., Chang, Z., Huang, Z., et al. (2018). Exosomes derived from miR-92a-3p-overexpressing human mesenchymal stem cells enhance chondrogenesis and suppress cartilage degradation via targeting WNT5A. Stem Cell Res. Ther. 9:247.

Martel-Pelletier, J., Barr, A. J., Cicuttini, F. M., Conaghan, P. G., Cooper, C., Goldring, M. B., et al. (2016). Osteoarthritis. Nat. Rev. Dis. Primers 2:16072.

Martin, J. A., and Buckwalter, J. A. (2002). Aging, articular cartilage chondrocyte senescence and osteoarthritis. Biogerontology 3, 257-264.

Mathiessen, A., and Conaghan, P. G. (2017). Synovitis in osteoarthritis: current understanding with therapeutic implications. Arthritis Res. Ther. 19:18.

McIntyre, J. A., Jones, I. A., Han, B., and Vangsness, C. T. Jr. (2018). Intra-articular mesenchymal stem cell therapy for the human joint: a systematic review. Am. J. Sports Med. 46, 3550-3563. doi: 10.1177/0363546517735844

Mendicino, M., Bailey, A. M., Wonnacott, K., Puri, R. K., and Bauer, S. R. (2014). MSC-based product characterization for clinical trials: an FDA perspective. Cell Stem Cell 14, 141-145. doi: 10.1016/j.stem.2014.01.013

Mesallati, T., Buckley, C. T., and Kelly, D. J. (2017). Engineering cartilaginous grafts using chondrocyte-laden hydrogels supported by a superficial layer of stem cells. J. Tissue Eng. Regen. Med. 11, 1343-1353. doi: 10.1002/term.2033

Mesallati, T., Sheehy, E. J., Vinardell, T., Buckley, C. T., and Kelly, D. J. (2015). Tissue engineering scaled-up, anatomically shaped osteochondral constructs for joint resurfacing. Eur. Cells Mater. 30, 163-185; discussion 85-86.

Mianehsaz, E., Mirzaei, H. R., Mahjoubin-Tehran, M., Rezaee, A., Sahebnasagh, R., Pourhanifeh, M. H., et al. (2019). Mesenchymal stem cell-derived exosomes: a new therapeutic approach to osteoarthritis? Stem Cell Res. Ther. 10:340.

Moradi, S., Mahdizadeh, H., Šarić, T., Kim, J., Harati, J., Shahsavarani, H., et al. (2019). Research and therapy with induced pluripotent stem cells (iPSCs): social, legal, and ethical considerations. Stem Cell Res. Ther. 10:341.

Mosser, D. M. (2003). The many faces of macrophage activation. J. Leukoc. Biol. 73, 209-212. doi: 10.1189/jlb.0602325

Mosser, D. M., and Edwards, J. P. (2008). Exploring the full spectrum of macrophage activation. Nat. Rev. Immunol. 8, 958-969. doi: 10.1038/nri2448

Murphy, C., Withrow, J., Hunter, M., Liu, Y., Tang, Y. L., Fulzele, S., et al. (2018). Emerging role of extracellular vesicles in musculoskeletal diseases. Mol. Aspects Med. 60, 123-128. doi: 10.1016/j.mam.2017.09.006

Murphy, M. B., Moncivais, K., and Caplan, A. I. (2013). Mesenchymal stem cells: environmentally responsive therapeutics for regenerative medicine. Exp. Mol. Med. 45:e54. doi: 10.1038/emm.2013.94

Nakagawa, T., Lee, S. Y., and Reddi, A. H. (2009). Induction of chondrogenesis from human embryonic stem cells without embryoid body formation by 
bone morphogenetic protein 7 and transforming growth factor betal. Arthritis Rheum. 60, 3686-3692. doi: 10.1002/art.27229

Nam, Y., Rim, Y. A., Lee, J., and Ju, J. H. (2018). Current therapeutic strategies for stem cell-based cartilage regeneration. Stem Cells Int. 2018:8490489.

Nejadnik, H., Diecke, S., Lenkov, O. D., Chapelin, F., Donig, J., Tong, X., et al. (2015). Improved approach for chondrogenic differentiation of human induced pluripotent stem cells. Stem Cell Rev. Rep. 11, 242-253. doi: 10.1007/s12015014-9581-5

Ni, Z., Kuang, L., Chen, H., Xie, Y., Zhang, B., Ouyang, J., et al. (2019). The exosome-like vesicles from osteoarthritic chondrocyte enhanced mature IL$1 \beta$ production of macrophages and aggravated synovitis in osteoarthritis. Cell Death Dis. 10:522.

Nims, R. J., Pferdehirt, L., Ho, N. B., Savadipour, A., Lorentz, J., Sohi, S., et al. (2021). A synthetic mechanogenetic gene circuit for autonomous drug delivery in engineered tissues. Sci. Adv. 7:eabd9858. doi: 10.1126/sciadv.abd9858

Oren, T. W., Botolin, S., Williams, A., Bucknell, A., and King, K. B. (2011). Arthroplasty in veterans: analysis of cartilage, bone, serum, and synovial fluid reveals differences and similarities in osteoarthritis with and without comorbid diabetes. J. Rehabil. Res. Dev. 48, 1195-1210. doi: 10.1682/jrrd.2010.09.0186

Ozeki, N., Muneta, T., Koga, H., Nakagawa, Y., Mizuno, M., Tsuji, K., et al. (2016). Not single but periodic injections of synovial mesenchymal stem cells maintain viable cells in knees and inhibit osteoarthritis progression in rats. Osteoarthritis Cartilage 24, 1061-1070. doi: 10.1016/j.joca.2015.12.018

Palazzo, C., Nguyen, C., Lefevre-Colau, M. M., Rannou, F., and Poiraudeau, S. (2016). Risk factors and burden of osteoarthritis. Ann. Phys. Rehabil. Med. 59, 134-138. doi: 10.1016/j.rehab.2016.01.006

Pap, T. K. A., and Bertrand, M. H. J. (2012). "Joint biochemistry," in Oxford Textbook of Rheumatology, 4th Edn, eds R. A. Watts, P. G. Conaghan, C. Denton, H. Foster, J. Isaacs, and U. Müller-Ladner (Oxford: Oxford University Press)

Pawitan, J. A., Bui, T. A., Mubarok, W., Antarianto, R. D., Nurhayati, R. W., Dilogo, I. H., et al. (2020). Enhancement of the therapeutic capacity of mesenchymal stem cells by genetic modification: a systematic review. Front. Cell Dev. Biol. 8:587776. doi: 10.3389/fcell.2020.587776

Pearson, M. J., Herndler-Brandstetter, D., Tariq, M. A., Nicholson, T. A., Philp, A. M., Smith, H. L., et al. (2017). IL-6 secretion in osteoarthritis patients is mediated by chondrocyte-synovial fibroblast cross-talk and is enhanced by obesity. Sci. Rep. 7:3451.

Peffers, M. J., Chabronova, A., Balaskas, P., Fang, Y., Dyer, P., Cremers, A., et al. (2020). SnoRNA signatures in cartilage ageing and osteoarthritis. Sci. Rep. 10:10641.

Pelttari, K., Winter, A., Steck, E., Goetzke, K., Hennig, T., Ochs, B. G., et al. (2006). Premature induction of hypertrophy during in vitro chondrogenesis of human mesenchymal stem cells correlates with calcification and vascular invasion after ectopic transplantation in SCID mice. Arthritis Rheum. 54, 3254-3266. doi: $10.1002 /$ art.22136

Pereira, D., Peleteiro, B., Araújo, J., Branco, J., Santos, R. A., and Ramos, E. (2011). The effect of osteoarthritis definition on prevalence and incidence estimates: a systematic review. Osteoarthritis Cartilage 19, 1270-1285. doi: 10.1016/j.joca. 2011.08.009

Pers, Y. M., Ruiz, M., Noël, D., and Jorgensen, C. (2015). Mesenchymal stem cells for the management of inflammation in osteoarthritis: state of the art and perspectives. Osteoarthritis Cartilage 23, 2027-2035. doi: 10.1016/j.joca.2015. 07.004

Pferdehirt, L., Ross, A. K., Brunger, J. M., and Guilak, F. (2019). A synthetic gene circuit for self-regulating delivery of biologic drugs in engineered tissues. Tissue Eng. Part A 25, 809-820. doi: 10.1089/ten.tea.2019.0027

Phinney, D. G., and Pittenger, M. F. (2017). Concise review: MSC-derived exosomes for cell-free therapy. Stem Cells (Dayton Ohio) 35, 851-858. doi: $10.1002 /$ stem. 2575

Pietschmann, P., Mechtcheriakova, D., Meshcheryakova, A., Föger-Samwald, U., and Ellinger, I. (2016). Immunology of Osteoporosis: a mini-review. Gerontology 62, 128-137. doi: 10.1159/000431091

Qi, H., Liu, D. P., Xiao, D. W., Tian, D. C., Su, Y. W., and Jin, S. F. (2019). Exosomes derived from mesenchymal stem cells inhibit mitochondrial dysfunctioninduced apoptosis of chondrocytes via p38, ERK, and Akt pathways. In Vitro Cell. Dev. Biol. Anim. 55, 203-210. doi: 10.1007/s11626-019-00330-x
Qu, C., Puttonen, K. A., Lindeberg, H., Ruponen, M., Hovatta, O., Koistinaho, J., et al. (2013). Chondrogenic differentiation of human pluripotent stem cells in chondrocyte co-culture. Int. J. Biochem. Cell Biol. 45, 1802-1812. doi: 10.1016/ j.biocel.2013.05.029

Rani, S., Ryan, A. E., Griffin, M. D., and Ritter, T. (2015). Mesenchymal stem cellderived extracellular vesicles: toward cell-free therapeutic applications. Mol. Ther. 23, 812-823. doi: 10.1038/mt.2015.44

Raposo, G., and Stoorvogel, W. (2013). Extracellular vesicles: exosomes, microvesicles, and friends. J. Cell Biol. 200, 373-383. doi: 10.1083/jcb. 201211138

Riegger, J., Palm, H. G., and Brenner, R. E. (2018). The functional role of chondrogenic stem/progenitor cells: novel evidence for immunomodulatory properties and regenerative potential after cartilage injury. Eur. Cells Mater. 36, 110-127. doi: 10.22203/ecm.v036a09

Rim, Y. A., Nam, Y., and Ju, J. H. (2020). The role of chondrocyte hypertrophy and senescence in osteoarthritis initiation and progression. Int. J. Mol. Sci. 21:2358. doi: 10.3390/ijms 21072358

Robinson, W. H., Lepus, C. M., Wang, Q., Raghu, H., Mao, R., Lindstrom, T. M., et al. (2016). Low-grade inflammation as a key mediator of the pathogenesis of osteoarthritis. Nat. Rev. Rheumatol. 12, 580-592. doi: 10.1038/nrrheum.2016. 136

Salminen, A., Kaarniranta, K., and Kauppinen, A. (2012). Inflammaging: disturbed interplay between autophagy and inflammasomes. Aging (Albany NY) 4, 166175. doi: 10.18632/aging.100444

Scanzello, C. R. (2017). Role of low-grade inflammation in osteoarthritis. Curr. Opin. Rheumatol. 29, 79-85. doi: 10.1097/bor.0000000000000353

Schubert, N., Dudeck, J., Liu, P., Karutz, A., Speier, S., Maurer, M., et al. (2015). Mast cell promotion of $\mathrm{T}$ cell-driven antigen-induced arthritis despite being dispensable for antibody-induced arthritis in which $\mathrm{T}$ cells are bypassed. Arthritis Rheumatol. 67, 903-913. doi: 10.1002/art. 38996

Scotti, C., Piccinini, E., Takizawa, H., Todorov, A., Bourgine, P., Papadimitropoulos, A., et al. (2013). Engineering of a functional bone organ through endochondral ossification. Proc. Natl. Acad. Sci. U.S.A. 110, 3997-4002. doi: 10.1073/pnas.1220108110

Sellam, J., and Berenbaum, F. (2010). The role of synovitis in pathophysiology and clinical symptoms of osteoarthritis. Nat. Rev. Rheumatol. 6, 625-635. doi: 10.1038/nrrheum.2010.159

Seol, D., McCabe, D. J., Choe, H., Zheng, H., Yu, Y., Jang, K., et al. (2012). Chondrogenic progenitor cells respond to cartilage injury. Arthritis Rheum. 64, 3626-3637. doi: 10.1002/art.34613

Shapiro, I. M., Landis, W. J., and Risbud, M. V. (2015). Matrix vesicles: are they anchored exosomes? Bone 79, 29-36. doi: 10.1016/j.bone.2015.05.013

Sharma, A. R., Jagga, S., Lee, S. S., and Nam, J. S. (2013). Interplay between cartilage and subchondral bone contributing to pathogenesis of osteoarthritis. Int. J. Mol. Sci. 14, 19805-19830. doi: 10.3390/ijms141019805

Shirasawa, S., Sekiya, I., Sakaguchi, Y., Yagishita, K., Ichinose, S., and Muneta, T. (2006). In vitro chondrogenesis of human synovium-derived mesenchymal stem cells: optimal condition and comparison with bone marrow-derived cells. J. Cell. Biochem. 97, 84-97. doi: 10.1002/jcb.20546

Shirinsky, I., and Shirinsky, V. (2018). H(1)-antihistamines are associated with lower prevalence of radiographic knee osteoarthritis: a cross-sectional analysis of the Osteoarthritis Initiative data. Arthritis Res. Ther. 20:116.

Siddappa, R., Licht, R., van Blitterswijk, C., and de Boer, J. (2007). Donor variation and loss of multipotency during in vitro expansion of human mesenchymal stem cells for bone tissue engineering. J. Orthop. Res. 25, 1029-1041. doi: 10.1002/jor.20402

Silverwood, V., Blagojevic-Bucknall, M., Jinks, C., Jordan, J. L., Protheroe, J., and Jordan, K. P. (2015). Current evidence on risk factors for knee osteoarthritis in older adults: a systematic review and meta-analysis. Osteoarthritis Cartilage 23, 507-515. doi: 10.1016/j.joca.2014.11.019

Singh, Y. P., Bandyopadhyay, A., and Mandal, B. B. (2019). 3D bioprinting using cross-linker-free silk-gelatin bioink for cartilage tissue engineering. ACS Appl. Mater. Interfaces 11, 33684-33696. doi: 10.1021/acsami.9b11644

Sophia Fox, A. J., Bedi, A., and Rodeo, S. A. (2009). The basic science of articular cartilage: structure, composition, and function. Sports Health 1, 461-468. doi: $10.1177 / 1941738109350438$ 
Sun, A. R., Friis, T., Sekar, S., Crawford, R., Xiao, Y., and Prasadam, I. (2016). Is synovial macrophage activation the inflammatory link between obesity and osteoarthritis? Curr. Rheumatol. Rep. 18:57.

Sun, A. R., Panchal, S. K., Friis, T., Sekar, S., Crawford, R., Brown, L., et al. (2017). Obesity-associated metabolic syndrome spontaneously induces infiltration of pro-inflammatory macrophage in synovium and promotes osteoarthritis. PLoS One 12:e0183693. doi: 10.1371/journal.pone.0183693

Sun, W., Zhao, C., Li, Y., Wang, L., Nie, G., Peng, J., et al. (2016). Osteoclastderived microRNA-containing exosomes selectively inhibit osteoblast activity. Cell Discov. 2:16015.

Sun, Y., Chen, S., and Pei, M. (2018). Comparative advantages of infrapatellar fat pad: an emerging stem cell source for regenerative medicine. Rheumatology (Oxford) 57, 2072-2086. doi: 10.1093/rheumatology/kex487

Takahashi, F., Takahashi, K., Shimizu, K., Cui, R., Tada, N., Takahashi, H., et al. (2004). Osteopontin is strongly expressed by alveolar macrophages in the lungs of acute respiratory distress syndrome. Lung 182, 173-185.

Takahashi, K., Tanabe, K., Ohnuki, M., Narita, M., Ichisaka, T., Tomoda, K., et al. (2007). Induction of pluripotent stem cells from adult human fibroblasts by defined factors. Cell 131, 861-872. doi: 10.1016/j.cell.2007.11.019

Tanaka, E., Aoyama, J., Miyauchi, M., Takata, T., Hanaoka, K., Iwabe, T., et al. (2005). Vascular endothelial growth factor plays an important autocrine/paracrine role in the progression of osteoarthritis. Histochem. Cell Biol. 123, 275-281. doi: 10.1007/s00418-005-0773-6

Taniguchi, N., Yoshida, K., Ito, T., Tsuda, M., Mishima, Y., Furumatsu, T., et al. (2007). Stage-specific secretion of HMGB1 in cartilage regulates endochondral ossification. Mol. Cell. Biol. 27, 5650-5663. doi: 10.1128/mcb.00130-07

Tao, S. C., Yuan, T., Zhang, Y. L., Yin, W. J., Guo, S. C., and Zhang, C. Q. (2017). Exosomes derived from miR-140-5p-overexpressing human synovial mesenchymal stem cells enhance cartilage tissue regeneration and prevent osteoarthritis of the knee in a rat model. Theranostics 7, 180-195. doi: 10.7150/ thno. 17133

Teitelbaum, S. L. (2000). Bone resorption by osteoclasts. Science 289, 1504-1508. doi: $10.1126 /$ science.289.5484.1504

Théry, C., Zitvogel, L., and Amigorena, S. (2002). Exosomes: composition, biogenesis and function. Nat. Rev. Immunol. 2, 569-579. doi: 10.1038/nri855

Thielen, N. G. M., van der Kraan, P. M., and van Caam, A. P. M. (2019). TGFB/BMP signaling pathway in cartilage homeostasis. Cells 8:969. doi: 10 . 3390/cells8090969

Thysen, S., Luyten, F. P., and Lories, R. J. (2015). Targets, models and challenges in osteoarthritis research. Dis. Models Mech. 8, 17-30. doi: 10.1242/dmm.016881

To, K., Zhang, B., Romain, K., Mak, C., and Khan, W. (2019). Synovium-Derived mesenchymal stem cell transplantation in cartilage regeneration: a PRISMA review of in vivo studies. Front. Bioeng. Biotechnol. 7:314. doi: 10.3389/fbioe. 2019.00314

Tofiño-Vian, M., Guillén, M. I., Pérez Del Caz, M. D., Castejón, M. A., and Alcaraz, M. J. (2017). Extracellular vesicles from adipose-derived mesenchymal stem cells downregulate senescence features in osteoarthritic osteoblasts. Oxid. Med. Cell. Longev. 2017:7197598.

Tofiño-Vian, M., Guillén, M. I., Pérez Del Caz, M. D., Silvestre, A., and Alcaraz, M. J. (2018). Microvesicles from human adipose tissue-derived mesenchymal stem cells as a new protective strategy in osteoarthritic chondrocytes. Cell. Physiol. Biochem. 47, 11-25. doi: 10.1159/000489739

Tonna, S., Poulton, I. J., Taykar, F., Ho, P. W., Tonkin, B., Crimeen-Irwin, B., et al. (2016). Chondrocytic ephrin B2 promotes cartilage destruction by osteoclasts in endochondral ossification. Development (Cambridge Engl.) 143, 648-657.

Tsuno, H., Suematsu, N., Sato, T., Arito, M., Matsui, T., Iizuka, N., et al. (2016). Effects of methotrexate and salazosulfapyridine on protein profiles of exosomes derived from a human synovial sarcoma cell line of SW982. Proteomics Clin. Appl. 10, 164-171. doi: 10.1002/prca.201500064

Urist, M. R., and McLean, F. C. (1957). Accumulation of mast cells in endosteum of bones of calcium-deficient rats. AMA Arch. Pathol. 63, 239-251.

Vina, E. R., and Kwoh, C. K. (2018). Epidemiology of osteoarthritis: literature update. Curr. Opin. Rheumatol. 30, 160-167. doi: 10.1097/bor. 0000000000000479

Visser, J. G., Van Staden, A. D. P., and Smith, C. (2019). Harnessing macrophages for controlled-release drug delivery: lessons from microbes. Front. Pharmacol. 10:22. doi: 10.3389/fphar.2019.00022
Vizoso, F. J., Eiro, N., Cid, S., Schneider, J., and Perez-Fernandez, R. (2017). Mesenchymal stem cell secretome: toward cell-free therapeutic strategies in regenerative medicine. Int. J. Mol. Sci. 18:1852. doi: 10.3390/ijms18091852

Vonk, L. A., van Dooremalen, S. F. J., Liv, N., Klumperman, J., Coffer, P. J., Saris, D. B. F., et al. (2018). Mesenchymal stromal/stem cell-derived extracellular vesicles promote human cartilage regeneration in vitro. Theranostics 8, 906920. doi: $10.7150 /$ thno. 20746

Wakitani, S., Imoto, K., Yamamoto, T., Saito, M., Murata, N., and Yoneda, M. (2002). Human autologous culture expanded bone marrow mesenchymal cell transplantation for repair of cartilage defects in osteoarthritic knees. Osteoarthritis Cartilage 10, 199-206. doi: 10.1053/joca.2001.0504

Wang, N., Liang, H., and Zen, K. (2014). Molecular mechanisms that influence the macrophage m1-m2 polarization balance. Front. Immunol. 5:614. doi: 10.3389/ fimmu.2014.00614

Wang, Q., Lepus, C. M., Raghu, H., Reber, L. L., Tsai, M. M., Wong, H. H., et al. (2019). IgE-mediated mast cell activation promotes inflammation and cartilage destruction in osteoarthritis. Elife 8:e39905.

Wang, R., Xu, B., and Xu, H. (2018). TGF- $\beta 1$ promoted chondrocyte proliferation by regulating $\mathrm{Sp} 1$ through MSC-exosomes derived miR-135b. Cell Cycle (Georgetown Tex) 17, 2756-2765. doi: 10.1080/15384101.2018.1556063

Wang, T., Wen, C. Y., Yan, C. H., Lu, W. W., and Chiu, K. Y. (2013). Spatial and temporal changes of subchondral bone proceed to microscopic articular cartilage degeneration in guinea pigs with spontaneous osteoarthritis. Osteoarthritis Cartilage 21, 574-581. doi: 10.1016/j.joca.2013.01.002

Wang, Y., Yu, D., Liu, Z., Zhou, F., Dai, J., Wu, B., et al. (2017). Exosomes from embryonic mesenchymal stem cells alleviate osteoarthritis through balancing synthesis and degradation of cartilage extracellular matrix. Stem Cell Res. Ther. 8:189.

Wei, Y., Zeng, W., Wan, R., Wang, J., Zhou, Q., Qiu, S., et al. (2012). Chondrogenic differentiation of induced pluripotent stem cells from osteoarthritic chondrocytes in alginate matrix. Eur. Cells Mater. 23, 1-12. doi: $10.22203 /$ ecm.v023a01

Wenham, C. Y., and Conaghan, P. G. (2010). The role of synovitis in osteoarthritis. Ther. Adv. Musculoskelet. Dis. 2, 349-359.

Willard, V. P., Diekman, B. O., Sanchez-Adams, J., Christoforou, N., Leong, K. W., and Guilak, F. (2014). Use of cartilage derived from murine induced pluripotent stem cells for osteoarthritis drug screening. Arthritis Rheumatol. 66, 3062-3072. doi: 10.1002/art.38780

Williams, R., Khan, I. M., Richardson, K., Nelson, L., McCarthy, H. E., Analbelsi, T., et al. (2010). Identification and clonal characterisation of a progenitor cell sub-population in normal human articular cartilage. PLoS One 5:e13246. doi: 10.1371/journal.pone.0013246

Woo, C. H., Kim, H. K., Jung, G. Y., Jung, Y. J., Lee, K. S., Yun, Y. E., et al. (2020). Small extracellular vesicles from human adipose-derived stem cells attenuate cartilage degeneration. J. Extracell. Vesicles 9:1735249. doi: 10.1080/20013078. 2020.1735249

Wu, C. L., Harasymowicz, N. S., Klimak, M. A., Collins, K. H., and Guilak, F. (2020). The role of macrophages in osteoarthritis and cartilage repair. Osteoarthritis Cartilage 28, 544-554. doi: 10.1016/j.joca.2019.12.007

Wu, J., Kuang, L., Chen, C., Yang, J., Zeng, W. N., Li, T., et al. (2019). miR100-5p-abundant exosomes derived from infrapatellar fat pad MSCs protect articular cartilage and ameliorate gait abnormalities via inhibition of mTOR in osteoarthritis. Biomaterials 206, 87-100. doi: 10.1016/j.biomaterials.2019.03. 022

Xie, J., Huang, Z., Yu, X., Zhou, L., and Pei, F. (2019). Clinical implications of macrophage dysfunction in the development of osteoarthritis of the knee. Cytokine Growth Factor Rev. 46, 36-44. doi: 10.1016/j.cytogfr.2019.03.004

Xue, Y. Z. B., Niu, Y. M., Tang, B., and Wang, C. M. (2019). PCL/EUG scaffolds with tunable stiffness can regulate macrophage secretion behavior. Prog. Biophys. Mol. Biol. 148, 4-11. doi: 10.1016/j.pbiomolbio.2019.05.006

Yamashita, A., Tamamura, Y., Morioka, M., Karagiannis, P., Shima, N., and Tsumaki, N. (2018). Considerations in hiPSC-derived cartilage for articular cartilage repair. Inflamm. Regen. 38:17.

Yan, L., and Wu, X. (2020). Exosomes produced from 3D cultures of umbilical cord mesenchymal stem cells in a hollow-fiber bioreactor show improved osteochondral regeneration activity. Cell Biol. Toxicol. 36, 165-178. doi: 10. 1007/s10565-019-09504-5 
Yokota, N., Hattori, M., Ohtsuru, T., Otsuji, M., Lyman, S., Shimomura, K., et al. (2019). Comparative clinical outcomes after intra-articular injection with adipose-derived cultured stem cells or noncultured stromal vascular fraction for the treatment of knee osteoarthritis. Am. J. Sports Med. 47, 2577-2583. doi: 10.1177/0363546519864359

Yu, J., Vodyanik, M. A., Smuga-Otto, K., Antosiewicz-Bourget, J., Frane, J. L., Tian, S., et al. (2007). Induced pluripotent stem cell lines derived from human somatic cells. Science 318, 1917-1920.

Yubo, M., Yanyan, L., Li, L., Tao, S., Bo, L., and Lin, C. (2017). Clinical efficacy and safety of mesenchymal stem cell transplantation for osteoarthritis treatment: a meta-analysis. PLoS One 12:e0175449. doi: 10.1371/journal.pone.017 5449

Zavatti, M., Beretti, F., Casciaro, F., Bertucci, E., and Maraldi, T. (2020). Comparison of the therapeutic effect of amniotic fluid stem cells and their exosomes on monoiodoacetate-induced animal model of osteoarthritis. BioFactors (Oxford Engl.) 46, 106-117. doi: 10.1002/biof. 1576

Zhang, R. K., Li, G. W., Zeng, C., Lin, C. X., Huang, L. S., Huang, G. X., et al. (2018). Mechanical stress contributes to osteoarthritis development through the activation of transforming growth factor beta 1 (TGF- $\beta 1$ ). Bone Joint Res. 7, 587-594. doi: 10.1302/2046-3758.711.bjr-2018-0057.r1

Zhang, S., Chu, W. C., Lai, R. C., Lim, S. K., Hui, J. H., and Toh, W. S. (2016). Exosomes derived from human embryonic mesenchymal stem cells promote osteochondral regeneration. Osteoarthritis Cartilage 24, 2135-2140. doi: 10. 1016/j.joca.2016.06.022

Zhang, S., Chuah, S. J., Lai, R. C., Hui, J. H. P., Lim, S. K., and Toh, W. S. (2018). MSC exosomes mediate cartilage repair by enhancing proliferation, attenuating apoptosis and modulating immune reactivity. Biomaterials 156, 16-27. doi: 10.1016/j.biomaterials.2017.11.028

Zhang, S., Teo, K. Y. W., Chuah, S. J., Lai, R. C., Lim, S. K., and Toh, W. S. (2019). MSC exosomes alleviate temporomandibular joint osteoarthritis by attenuating inflammation and restoring matrix homeostasis. Biomaterials 200, 35-47. doi: 10.1016/j.biomaterials.2019.02.006

Zhang, Y., and Jordan, J. M. (2008). Epidemiology of osteoarthritis. Rheum. Dis. Clin. North Am. 34, 515-529.

Zhao, C., Chen, J. Y., Peng, W. M., Yuan, B., Bi, Q., and Xu, Y. J. (2020). Exosomes from adipose-derived stem cells promote chondrogenesis and suppress inflammation by upregulating miR-145 and miR-221. Mol. Med. Rep. 21, 1881-1889.

Zhao, Y., and Xu, J. (2018). Synovial fluid-derived exosomal lncRNA PCGEM1 as biomarker for the different stages of osteoarthritis. Int. Orthop. 42, 2865-2872. doi: 10.1007/s00264-018-4093-6

Zhao, Y., Liu, H., Zhao, C., Dang, P., Li, H., and Farzaneh, M. (2020). Paracrine interactions involved in human induced pluripotent stem cells differentiation into chondrocytes. Curr. Stem Cell Res. Ther. 15, 233-242. doi: 10.2174/ $1574888 \times 15666191224122058$

Zhen, G., Wen, C., Jia, X., Li, Y., Crane, J. L., Mears, S. C., et al. (2013). Inhibition of TGF- $\beta$ signaling in mesenchymal stem cells of subchondral bone attenuates osteoarthritis. Nat. Med. 19, 704-712. doi: 10.1038/nm.3143

Zheng, L., Wang, Y., Qiu, P., Xia, C., Fang, Y., Mei, S., et al. (2019). Primary chondrocyte exosomes mediate osteoarthritis progression by regulating mitochondrion and immune reactivity. Nanomedicine (Lond. Engl.) 14, 31933212. doi: $10.2217 / \mathrm{nnm}-2018-0498$

Zhu, Y., Wang, Y., Zhao, B., Niu, X., Hu, B., Li, Q., et al. (2017). Comparison of exosomes secreted by induced pluripotent stem cell-derived mesenchymal stem cells and synovial membrane-derived mesenchymal stem cells for the treatment of osteoarthritis. Stem Cell Res. Ther. 8:64.

Conflict of Interest: The authors declare that the research was conducted in the absence of any commercial or financial relationships that could be construed as a potential conflict of interest.

Publisher's Note: All claims expressed in this article are solely those of the authors and do not necessarily represent those of their affiliated organizations, or those of the publisher, the editors and the reviewers. Any product that may be evaluated in this article, or claim that may be made by its manufacturer, is not guaranteed or endorsed by the publisher.

Copyright (C) 2021 Li, Huang and Bai. This is an open-access article distributed under the terms of the Creative Commons Attribution License (CC BY). The use, distribution or reproduction in other forums is permitted, provided the original author(s) and the copyright owner(s) are credited and that the original publication in this journal is cited, in accordance with accepted academic practice. No use, distribution or reproduction is permitted which does not comply with these terms. 